\title{
ChREBP regulates fructose-induced glucose production independently of insulin signaling
}

\author{
Mi-Sung Kim, ${ }^{1}$ Sarah A. Krawczyk, ${ }^{1}$ Ludivine Doridot, ${ }^{1}$ Alan J. Fowler, ${ }^{1}$ Jennifer X. Wang, ${ }^{2}$ Sunia A. Trauger, ${ }^{2}$ Hye-Lim Noh, ${ }^{3}$ \\ Hee Joon Kang, ${ }^{3}$ John K. Meissen, ${ }^{4}$ Matthew Blatnik, ${ }^{4}$ Jason K. Kim, ${ }^{3}$ Michelle Lai, ${ }^{5}$ and Mark A. Herman ${ }^{1,6}$ \\ 'Division of Endocrinology, Beth Israel Deaconess Medical Center, Boston, Massachusetts, USA. ²Faculty of Arts and Sciences, Harvard University, Cambridge, Massachusetts, USA. ${ }^{3}$ Program in Molecular Medicine, \\ University of Massachusetts Medical School, Worcester, Massachusetts, USA. ${ }^{4}$ Department of Pharmacokinetics, Dynamics and Metabolism, Pfizer Global Research and Development, Groton, Connecticut, USA. \\ ${ }^{5}$ Division of Gastroenterology, Beth Israel Deaconess Medical Center, Boston, Massachusetts, USA. ${ }^{6}$ Broad Institute, Cambridge, Massachusetts, USA.
}

\begin{abstract}
Obese, insulin-resistant states are characterized by a paradoxical pathogenic condition in which the liver appears to be selectively insulin resistant. Specifically, insulin fails to suppress glucose production, yet successfully stimulates de novo lipogenesis. The mechanisms underlying this dysregulation remain controversial. Here, we hypothesized that carbohydrateresponsive element-binding protein (ChREBP), a transcriptional activator of glycolytic and lipogenic genes, plays a central role in this paradox. Administration of fructose increased hepatic hexose-phosphate levels, activated ChREBP, and caused glucose intolerance, hyperinsulinemia, hypertriglyceridemia, and hepatic steatosis in mice. Activation of ChREBP was required for the increased expression of glycolytic and lipogenic genes as well as glucose-6-phosphatase (G6pc) that was associated with the effects of fructose administration. We found that fructose-induced G6PC activity is a major determinant of hepatic glucose production and reduces hepatic glucose-6-phosphate levels to complete a homeostatic loop. Moreover, fructose activated ChREBP and induced G6pc in the absence of Foxo1a, indicating that carbohydrate-induced activation of ChREBP and G6PC dominates over the suppressive effects of insulin to enhance glucose production. This ChREBP/C6PC signaling axis is conserved in humans. Together, these findings support a carbohydrate-mediated, ChREBP-driven mechanism that contributes to hepatic insulin resistance.
\end{abstract}

\section{Introduction}

The metabolic syndrome is a cluster of disorders including obesity, nonalcoholic fatty liver disease (NAFLD), hypertriglyceridemia, and insulin resistance, which predisposes to the development of type 2 diabetes and cardiovascular disease (1). This syndrome is also characterized by a pathogenic paradox in which the liver appears selectively insulin resistant: hyperinsulinemia is unable to appropriately suppress glucose production, but the liver remains sensitive to insulin's ability to stimulate de novo lipogenesis (DNL) (2).

Despite extensive investigation, the mechanisms accounting for this apparent paradox remain controversial. Investigation at the level of hepatic insulin signaling suggested that different arms of the insulin signaling cascade might be differentially sensitive to insulin (3-6). For instance, insulin signaling to inhibit the transcription factor forkhead box O1a (FOXO1A), which transactivates gluconeogenic enzyme gene expression, might be impaired, while insulin-mediated stimulation of a mechanistic target of rapamycin (MTOR)/sterol regulatory element-binding transcription factor 1 (SREBPF1 or SREBP1) signaling axis, which upregulates a DNL gene program, remains intact (5). However, others reported that defects in hepatic insulin signaling occur at the level of the insulin recep-

Authorship note: M.S. Kim and S.A. Krawczyk contributed equally to this work Conflict of interest: J.K. Meissen and M. Blatnik are employees of Pfizer Inc. Submitted: March 23, 2015; Accepted: August 18, 2016.

Reference information: J Clin Invest. 2016;126(11):4372-4386. doi:10.1172/JCI81993. tor, proximal to the proposed branch points in insulin signaling, thereby precluding differential insulin signaling as a mechanism to explain this paradox (7). It has also been suggested that insulin's acute effect of reducing hepatic glucose production (HGP) is independent of hepatic insulin signaling altogether and instead depends on suppression of adipose lipolysis and delivery of fatty-acid substrate to the liver $(8,9)$. However, this contrasts with other studies indicating that insulin's acute effect of reducing glucose production is mediated through insulin's actions directly on the liver $(10,11)$. These studies do not address the observation that hyperinsulinemia fails to suppress hepatic glucose-6-phosphatase (G6PC) expression in insulin-resistant humans (12), a condition where increased DNL in association with steatosis and hypertriglyceridemia is readily apparent $(13,14)$. Understanding the signaling mechanism regulating G6pc in this context is important, as increased hepatic G6PC is sufficient to cause glucose intolerance and insulin resistance (15).

Another mechanism that may contribute to the paradox is that glucose or gluconeogenic substrate delivered to the liver can activate hepatic DNL independently of insulin signaling (16-19). Carbohydrate-responsive element-binding protein (ChREBP, also known as MLXIPL) is a master transcriptional regulator of glycolytic and lipogenic genes and is activated by carbohydrate $(\mathrm{CHO})$ metabolites in key metabolic tissues including liver. In insulin-resistant states, when glucose disposal is impaired in peripheral tissues such as skeletal muscle and adipose tissue, shunting of glucose to the liver might activate ChREBP and enhance DNL. For instance, genetically ablating Glut4, the insulin-responsive glucose transport- 

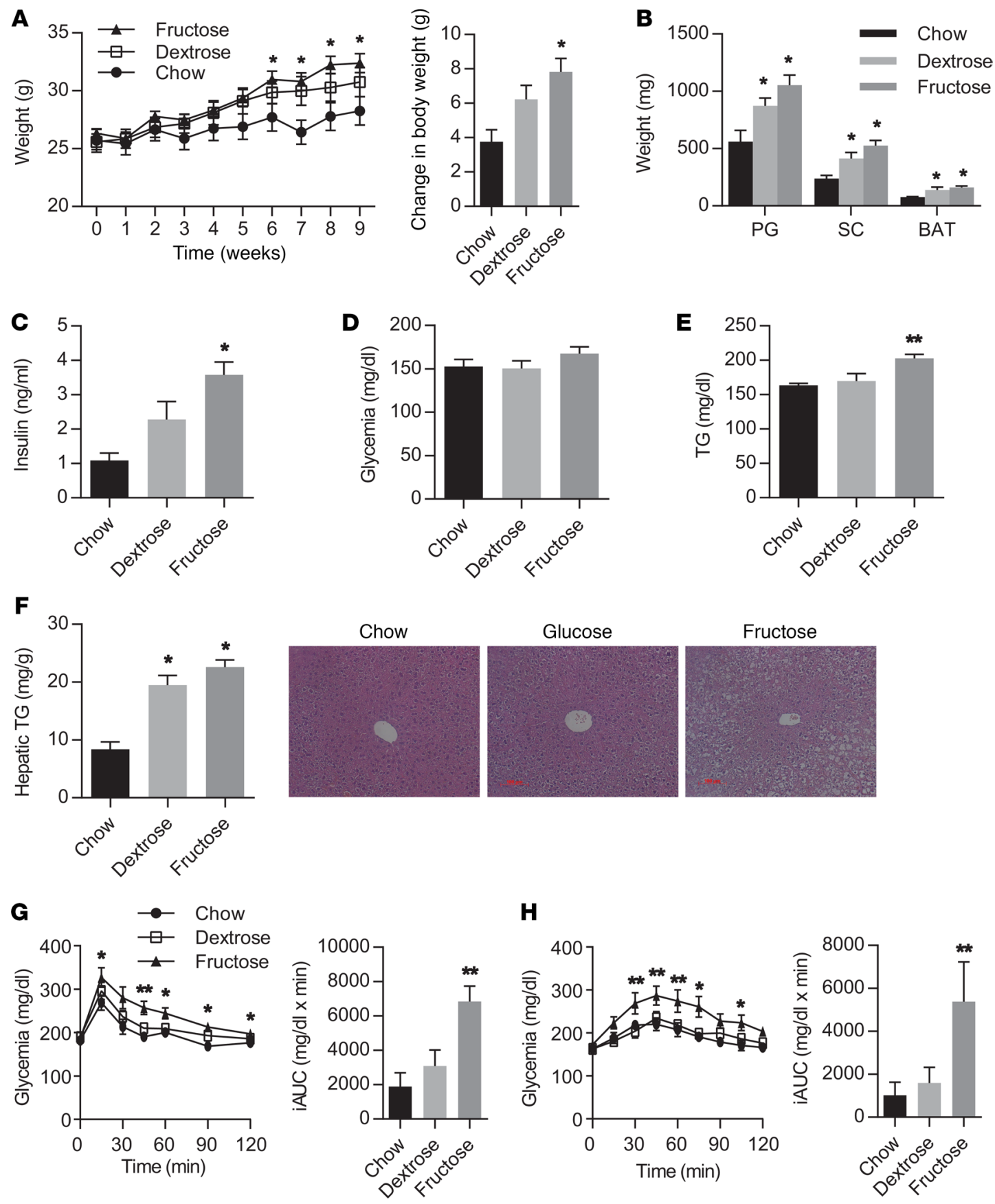

Figure 1. High-fructose feeding induces metabolic disease. (A) Body weight and (B) fat pad weights (PG, perigonadal; SC, subcutaneous; BAT, brown adipose tissue) were measured in WT male mice fed chow, high-dextrose, or high-fructose diet for 9 weeks $(n=8$ per group). (C) Glycemia and (D) serum insulin were measured in the ad libitum-fed state. (E) Serum triglyceride (TC) levels were measured after an overnight fast followed by 3 hours refeeding with chow, glucose, or fructose diet. (F) Hepatic TC levels and representative H\&E-stained liver sections. Images were obtained at $\times 20$ magnification. (G) Glucose tolerance test and (H) glycerol tolerance test with incremental areas under the curve ( $\triangle \mathrm{AUC}$ ) for this cohort. $P$ values were obtained by 1-way ANOVA. ${ }^{*} P<0.05$ versus chow; ${ }^{* *} P<0.05$ versus all others. Values are the mean $\pm \mathrm{SEM}$.

er, from skeletal muscle and adipose tissue enhances hepatic DNL (20). Additionally, siRNA-mediated knockdown of ChREBP in $o b / o b$ mice decreases hepatic DNL in the setting of persistent hyperinsulinemia (21). Thus, hepatic DNL may be regulated by increased substrate delivery independently of insulin signaling. However, whether increasing intrahepatic $\mathrm{CHO}$ metabolites might also signal to increase glucose production has not been fully explored.
It has recently been hypothesized that hepatocyte $\mathrm{CHO}$ metabolites (hexose- or triose-phosphates) might not only stimulate ChREBP and activate DNL, but might also serve as a signal to enhance HGP $(17,18)$. This hypothesis derives in part from the counterintuitive observation that while ChREBP is known to stimulate glycolysis through transactivation of glycolytic genes (22), it may also transactivate expression of $G 6 p c$ encoding the enzyme 
A
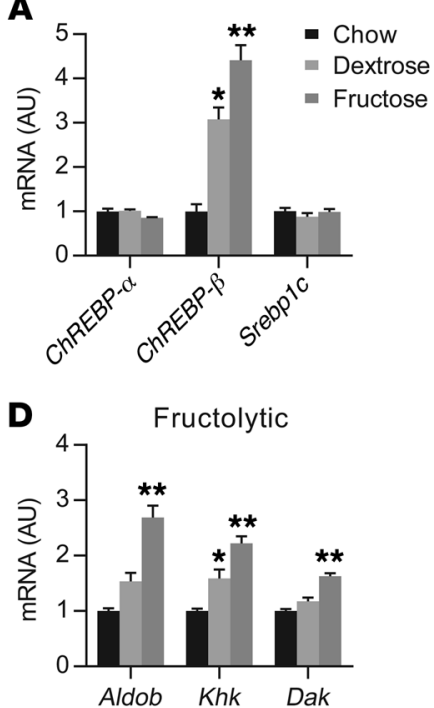

B

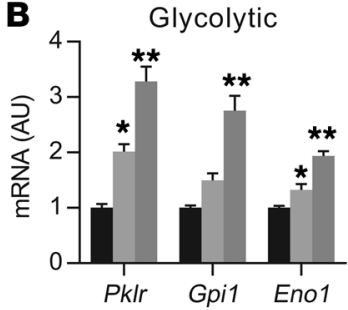

E

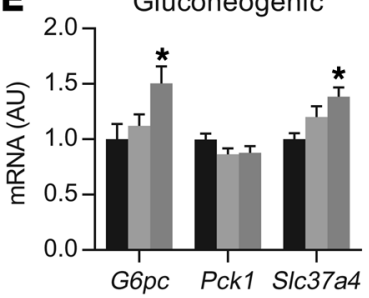

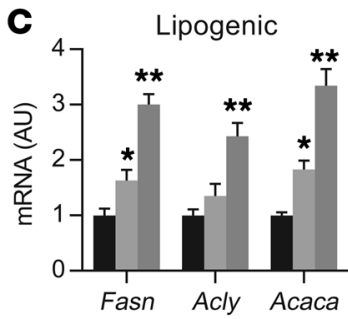

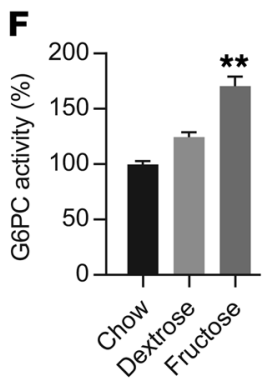

Figure 2. High-fructose feeding activates hepatic ChREBP and its metabolic gene targets. WT male mice were ad libitum fed a chow, high-dextrose, or high-fructose diet for 9 weeks ( $n=8$ per group) and (A-E) hepatic gene expression and (F) hepatic G6PC activity were measured. $P$ values were obtained by 1 -way ANOVA. ${ }^{*} P<0.05$ versus chow diet; ${ }^{* *} P<0.05$ versus all others. Values are the mean \pm SEM catalyzing the final step in glucose production $(23,24)$. Consistent with this, G6pc expression is diminished in $o b / o b$ mice when ChREBP is knocked down or crossed with ChREBP knockout (ChKO) mice $(21,25)$. Here, we test whether HGP may be regulated by the abundance of hepatic substrate and whether ChREBP is a key mechanistic link in this process.

We administered fructose to mice to model the effects of increased hepatic CHO metabolites, since fructose is preferentially metabolized in the liver (26) and can rapidly increase hepatic $\mathrm{CHO}$ metabolite levels (27). Also, increased fructose consumption as a component of sucrose or high-fructose corn syrup is increasingly associated with cardiometabolic disease in humans (28). Excessive fructose consumption in both humans and rodents can produce or exacerbate all of the features of metabolic syndrome including increased fatty liver, hepatic de novo lipogenesis, and increased glucose production $(29,30)$. Recently, Schwarz and colleagues demonstrated that a weight-maintaining high-fructose diet causes increased hepatic DNL and hepatic insulin resistance within 1 week (31). Thus, even short-term, weight-maintaining fructose administration can reproduce the pathophysiology associated with the pathogenic paradox of hepatic insulin resistance.

ChREBP plays an important role in fructose metabolism and fructose-induced metabolic disease, as it has been shown that whole-body ChKO mice are intolerant to diets containing fructose (22) and knockdown of ChREBP selectively in liver and adipose tissue of high-fructose-fed rats reduces DNL and hypertriglyceridemia and improves peripheral insulin sensitivity (32). A role for ChREBP in HGP has not been extensively examined.

\section{Results}

High-fructose feeding activates hepatic ChREBP and induces metabolic disease. High-fructose feeding has been reported to activate hepatic ChREBP and its transcriptional targets in rodents (32-35). We recently discovered a potentially novel potent isoform of $C h R E B P, C h R E B P-\beta$, which is expressed from an alternative promoter in the ChREBP gene (36). To determine whether fructose might stimulate hepatic expression of ChREBP- $\beta$ in association with the development of metabolic disease, we fed mice chow, $60 \%$ dextrose (HDD), or 60\% fructose (HFrD) diets for 9 weeks. HFrD induced a significant increase in body weight compared with chow, with a trend toward increased body weight on HDD (Figure 1A). Adiposity was significantly increased in both HFrD and HDD compared with chow (Figure 1B). Ad libitum-fed insulin levels were significantly higher in HFrD (Figure 1C) compared with chow, without differences in blood glucose levels (Figure 1D), suggesting that fructose-fed mice are insulin resistant. HFrD, but not HDD, significantly increased plasma triglyceride (Figure 1E). Both HDD and HFrD significantly increased hepatic steatosis (Figure 1F). HFrD, but not HDD, caused glucose intolerance (Figure 1G) and increased glycemic excursion following glycerol injection (Figure 1H): a glycerol tolerance test. Together, these results demonstrate that increased fructose consumption can induce metabolic disease including impaired glucose and lipid homeostasis compared with chow-fed mice and independently of obesity, adiposity, and hepatic steatosis when compared with dextrose-fed mice.

We next examined whether ChREBP might be involved in HFrD-induced metabolic disease by measuring hepatic gene expression of ChREBP and Srebp1c - another important transcriptional regulator of hepatic DNL (37) - and the expression of glycolytic, fructolytic, lipogenic, and gluconeogenic enzymes (Figure 2, A-E). HFrD markedly induced hepatic ChREBP- $\beta$, but not ChREBP- $\alpha$ or Srebp1c (Figure 2A). The relative importance of ChREBP- $\alpha$ versus $-\beta$ in terms of total ChREBP activity is currently unknown. However, because ChREBP- $\beta$ expression requires ChREBP- $\alpha$ activity and ChREBP- $\beta$ is more transcriptionally potent than ChREBP- $\alpha, C h R E B P-\beta$ expression is the best-known surrogate marker of total ChREBP activity (36). Increased ChREBP activity with HFrD was accompanied by increased glycolytic (liver pyruvate kinase [Pklr], glucose phosphate isomerase [Gpi1], enolase 1[Eno1]), fructolytic (aldolase B [Aldob], ketohexokinase [Khk], dihydroxyacetone kinase $[D a k]$ ), and lipogenic (fatty acid synthase [Fasn], 
A

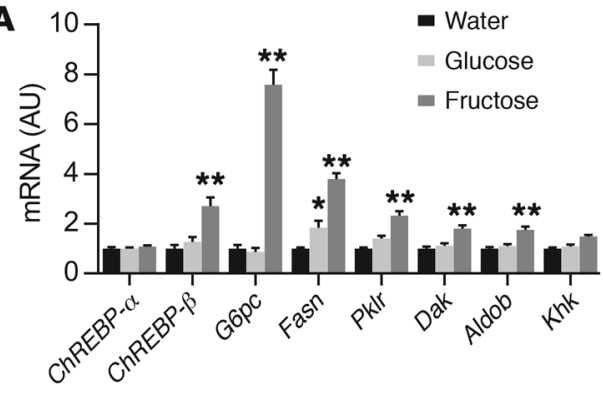

C
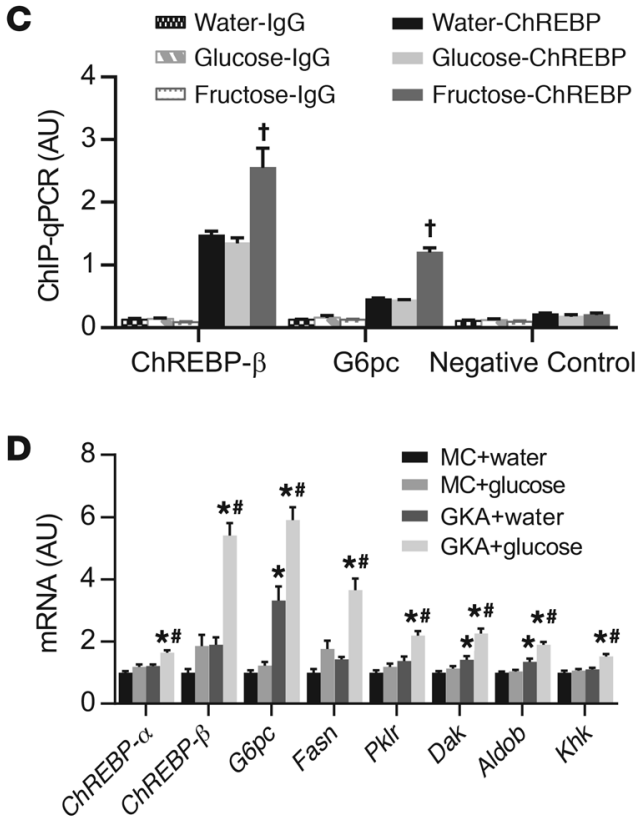

B
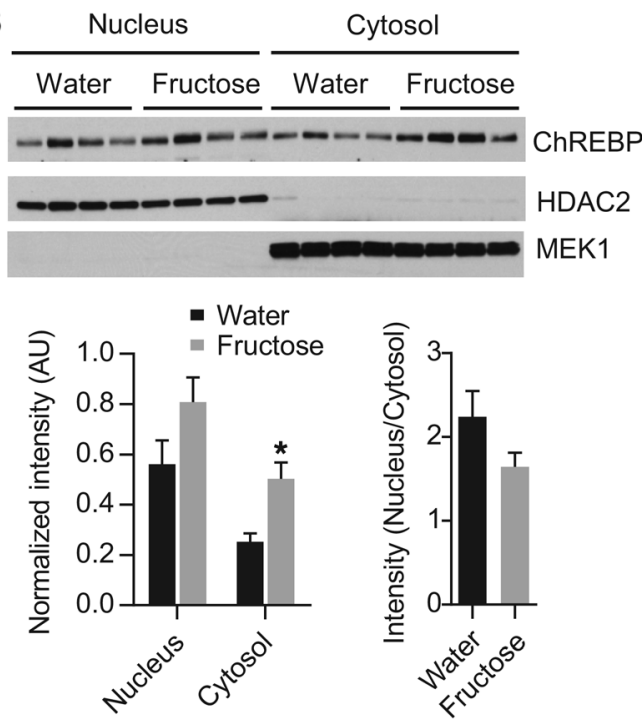

$\mathbf{E}$

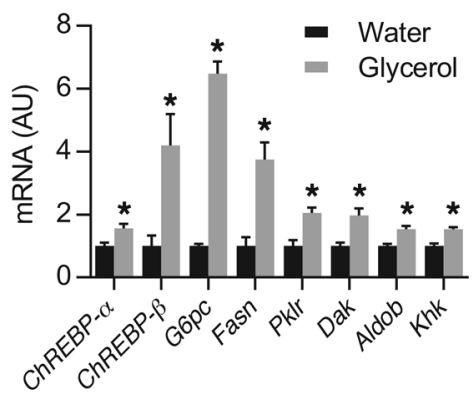

Figure 3. Multiple different carbohydrates acutely activate hepatic ChREBP. (A-C) Five-hour-fasted, 8-week-old C3H/HeJ male mice were gavaged with water, glucose ( $4 \mathrm{~g} / \mathrm{kg}$ body weight), or fructose $(4 \mathrm{~g} / \mathrm{kg}$ body weight) and sacrificed 100 minutes later. (A) Hepatic gene expression was measured by qPCR. $P$ values were obtained by 1-way ANOVA. ${ }^{*} P<0.05$ compared with water; ${ }^{* *} P<0.05$ compared with all others $(n=6$ per group). (B) Nuclear and cytosolic ChREBP- $\alpha$ and the ratio of nuclear to cytosolic ChREBP- $\alpha$ from water- versus fructose-gavaged mice were measured by Western blot and quantified ( $n=4$ per group). $P$ values were obtained by Student's $t$ test. ${ }^{*} P<0.05$ compared with water. (C) ChIP was performed from liver tissue with anti-ChREBP antibody or IgG control and qPCR was performed on immunoprecipitated chromatin with primers spanning the E-box in the ChREBP- $\beta$ promoter, the carbohydrate response element in the G6pc promoter, and a nonspecific genomic region. $P$ values were obtained by 1 -way ANOVA. ${ }^{\dagger} P<0.05$ compared with water- and glucose-ChREBP groups ( $n=4$ per group). (D) Five-hour-fasted, 8-week-old C3H/HeJ male mice were gavaged with glucokinase activator (PF-04991532, $100 \mathrm{mg} / \mathrm{kg}$ body weight) and then gavaged with water or glucose (4 g/ $/ \mathrm{kg}$ body weight) 30 minutes later. Mice were sacrificed 100 minutes later and hepatic gene expression was measured by qPCR. $P$ values were obtained by 2 -way ANOVA. ${ }^{*} P<0.05$ compared with methylcellulose (MC) within water- or glucose-gavaged groups; $\# P<0.05$ compared with water within MC- or GKA-gavaged groups ( $n=6$ per group). (E) Five-hour-fasted, 8-week-old $\mathrm{C} 3 \mathrm{H} / \mathrm{HeJ}$ male mice were gavaged with water or glycerol $(4 \mathrm{~g} / \mathrm{kg}$ body weight) and sacrificed 100 minutes later. $P$ values were obtained by Student's $t$ test. ${ }^{*} P<0.05$ compared with water $(n=6$ per group). Values are the mean \pm SEM.

ATP-citrate lyase $[A c l y]$, acetyl-CoA carboxylase- $\alpha[$ Acaca] $)$ gene expression compared with HDD and chow. Additionally, HFrD, but not HDD, induced expression of factors essential for glucose production including G6pc and Slc37a4, which mediates transport of glucose-6-phosphate (G6P) into the endoplasmic reticulum lumen where G6PC catalytic activity resides. In contrast, expression of the gluconeogenic enzyme phosphoenolpyruvate carboxykinase (Pck1) was affected by neither HFrD nor HDD, indicating that the effects of fructose and potentially ChREBP on glucose production may be selective for different portions of the gluconeogenic pathway.

Consistent with gene expression data, G6PC enzymatic activity was significantly increased on HFrD, but not HDD (Figure 2F), suggesting that HFrD may induce features of metabolic disease by activating hepatic ChREBP and increasing hepatic DNL and glucose production via upregulation of lipogenic and gluconeogenic gene programs, respectively.

CHOs metabolized by the liver acutely activate ChREBP. We next planned to test whether ChREBP is essential for the fructose-induced changes in gene expression using ChKO mice. However, because ChKO mice are intolerant to dietary fructose and cease eating when challenged with fructose-containing diets (ref. 22 and Supplemental Figure 1; supplemental material available online with this article; doi:10.1172/JCI81993DS1), we first established a gavage paradigm to deliver defined amounts of different $\mathrm{CHO}$ and examine their acute effects on hepatic gene expression. Fructose is largely extracted by the liver first pass (26). In contrast, 
A
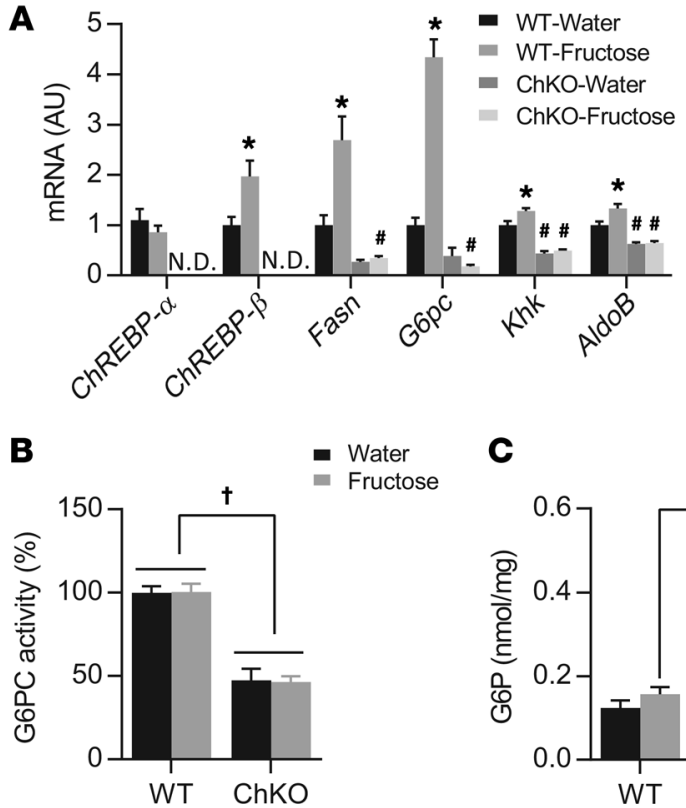

- Water

C

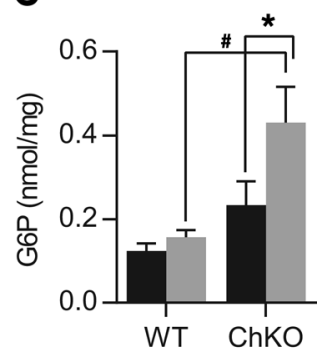

orally ingested glucose largely escapes hepatic first-pass metabolism (38). This occurs in part because glucokinase (GCK), which catalyzes the committed step in hepatic glucose metabolism, is sequestered and inactivated in the nucleus under the control of the GCK regulatory protein (GCKR) (39). We hypothesized that fructose gavage would more robustly activate ChREBP and its targets compared with glucose. Indeed, fructose gavage, but not glucose gavage, acutely increased the expression of hepatic ChREBP- $\beta$ 2.7fold $(P<0.001)$ (Figure 3A). This was accompanied by increased expression of glycolytic, fructolytic, and lipogenic genes as well as a robust, 7 -fold increase in $G 6 p c(P<0.0001)$ (Figure 3A).

The mechanisms by which CHO metabolites activate ChREBP- $\alpha$ to increase expression of ChREBP- $\beta$ and other ChREBP transcriptional targets remains controversial (reviewed in ref. 40). Nuclear translocation of ChREBP- $\alpha$ may be important for CHO-mediated activation (41-43). However, ChREBP- $\alpha$ remains predominantly cytosolic in low- and high-CHO conditions and the transcriptional activity of mutant forms of ChREBP- $\alpha$, which are constitutively localized to the nucleus, remain glucose responsive, indicating that the ability of glucose metabolites to stimulate ChREBP- $\alpha$ transcrip-

Figure 5. ChREBP mediates the conversion of fructose to glucose. We measured accumulation of glucose in the media after treatment with fructose or lactate+pyruvate from mouse primary hepatocytes obtained from (A) WT mice fed chow versus HFrD for 1 week ( $n=3$ per group) and (B) chow-fed WT versus ChKO mice ( $n=4$ per group). $P$ values were obtained by 2-way ANOVA. ${ }^{*} P<0.05$ compared with lactate+pyruvate within genotype or diet; ${ }^{\#} P<0.05$ compared with lactate+pyruvate condition within genotype or diet. (C) Serum fructose concentrations were determined by LC-MS at the indicated time points in 5-hour-fasted, 8- to 12-week-old WT and ChKO male mice after gavage with U13C-fructose ( $4 \mathrm{~g} / \mathrm{kg}$ body weight). $P$ values were obtained by Student's $t$ test. ${ }^{*} P<0.05$ compared with WT ( $n=5$ per group). (D) A schematic diagram illustrating our working hypothesis regarding how ChREBP regulates intracellular hexose-phosphate homeostasis. Increased sugar consumption increases hepatic carbohydrate uptake, which activates ChREBP and increases glycolysis, fatty acid synthesis, and glucose production to dispose of hexose-phosphates such as G6P.

Figure 4. ChREBP is necessary for fructose-induced hepatic gene expression. (A) Hepatic gene expression, (B) hepatic G6PC activity, and (C) hepatic G6P levels were measured in 5-hour-fasted, 8- to 12-week-old WT and ChKO male mice gavaged with water or fructose $(4 \mathrm{~g} / \mathrm{kg}$ body weight) and sacrificed 100 minutes later ( $n=6-9$ per group). $P$ values were obtained by 2-way ANOVA. ${ }^{*} P<0.05$ compared with water within genotype; ${ }^{\#} P<0.05$ compared with WT within gavage treatment; ${ }^{\dagger} P<0.05$ main effect of genotype. Values are the mean \pm SEM.

tional activity is likely independent of enhanced nuclear import $(44,45)$. As the majority of these studies have been conducted in vitro, we evaluated aspects of these models in our in vivo gavage paradigm. Western blots were performed in nuclear versus cytosolic fractions from water- versus fructose-gavaged mice (Figure $3 B)$. ChREBP- $\alpha$ protein was readily detectable in both subcellular fractions. ChREBP- $\beta$ protein was not detectable. This may be due to low absolute ChREBP- $\beta$ expression levels and rapid ChREBP- $\beta$ degradation, as suggested by our prior results (36). Despite the fact that $C h R E B P-\alpha$ gene expression did not change with fructose gavage (Figure 3A), the abundance of ChREBP- $\alpha$ protein in the cytosol increased, suggesting that fructose may enhance ChREBP- $\alpha$ stability. A tendency towards increased ChREBP- $\alpha$ protein was also observed in the nuclear fraction, though this did not achieve statistical significance. As a result, the nuclear to cytosolic ChREBP- $\alpha$ ratio actually tended to decrease with fructose gavage. This suggests that enhancing ChREBP- $\alpha$ nuclear import is not a major mechanism by which $\mathrm{CHO}$ metabolites activate ChREBP in vivo. To further assess mechanisms by which CHO might activate ChREBP, we assessed whether the fructose-mediated increases in ChREBP- $\beta$ and G6pc expression were associated with increased ChREBP binding at $\mathrm{CHO}$ response elements within their promoters by chromatin immunoprecipitation coupled with quantitative PCR (ChIP-qPCR) (Figure 3C). We observed robust ChREBP binding to its genomic targets in basal conditions and this increased 2- to 3-fold with fructose gavage. This increase in ChREBP binding to genomic targets occurred without a corresponding increase in nuclear ChREBP protein (Figure

\section{A}
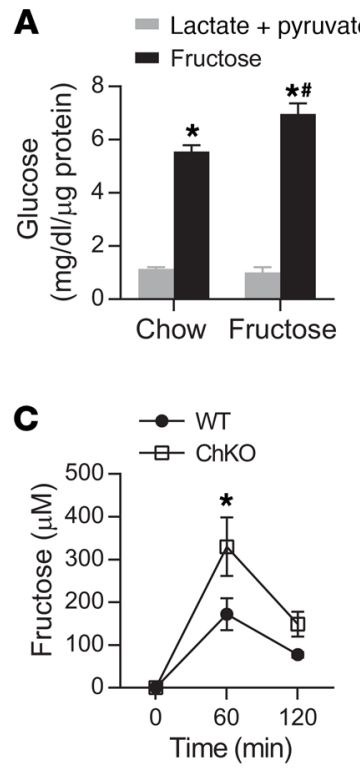

B
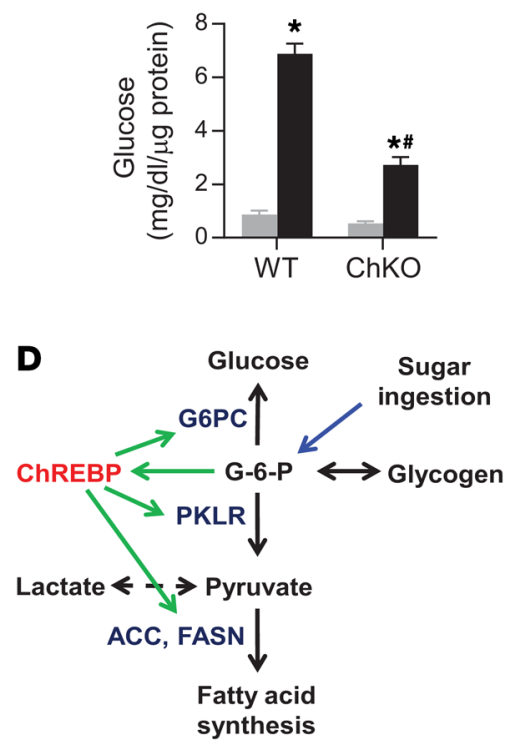
A

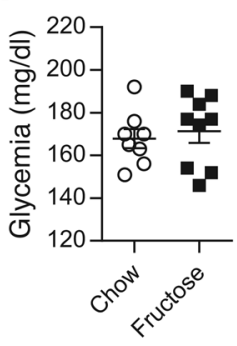

B

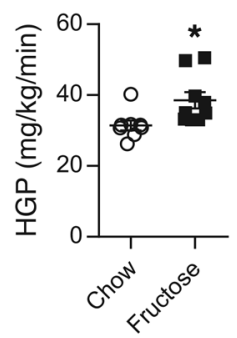

C

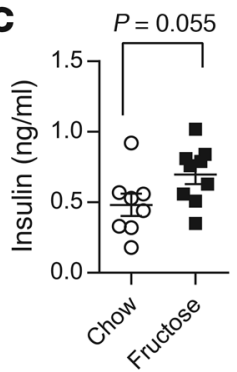

D

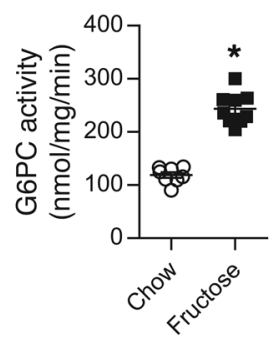

E

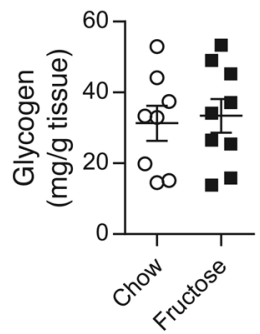

$\mathbf{F}$

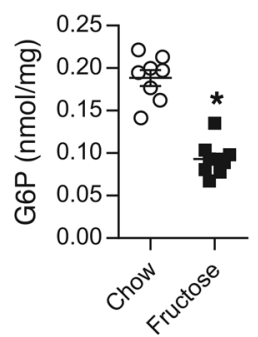

G

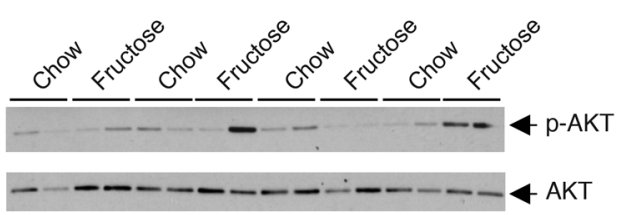

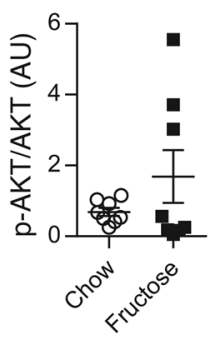

H

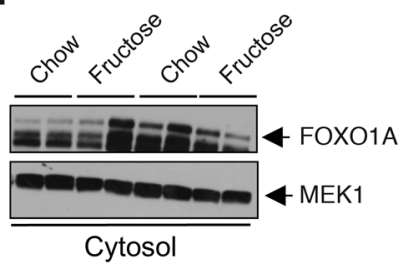

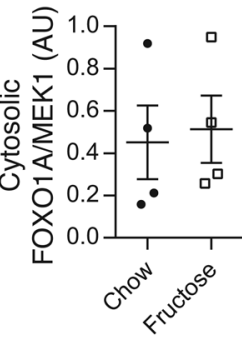

Figure 6. HFrD increases HGP in association with increased G6PC activity. (A) Glycemia (B) HCP, and (C) serum insulin levels were measured after a 4-hour fast in 4-month-old live, conscious mice fed chow vs. HFrD for 2 weeks ( $n=8$ or 9 per group). (D) Hepatic G6PC activity, (E) hepatic glycogen, and (F) hepatic G6P levels were measured in liver obtained from mice euthanized immediately after blood was obtained for the turnover measurement. $P$ values were obtained by Student's $t$ test. ${ }^{*} P<0.05$ compared with chow. (C) Western blot of hepatic total AKT and phospho-AKT (Ser473), with quantification of the AKT/phospo-AKT ratio ( $n=8$ per group). (H) Western blot of hepatic cytosolic FOXO1A with quantification of FOXO1A normalized for MEK1 $(n=4$ per group). Bars represent the mean $\pm \mathrm{SEM}$.

3B). These results are consistent with a model in which sufficient ChREBP protein is constitutively present in hepatic nuclei. Some of this nuclear ChREBP is bound to its genomic targets in basal conditions. Under the influence of increased $\mathrm{CHO}$ metabolites, binding of ChREBP to its targets is further enhanced.

To test whether the acute activation of hepatic ChREBP was mediated by a fructose-specific metabolite or whether other $\mathrm{CHO}$ metabolites could also activate hepatic ChREBP, we tested whether a liver-selective GCK activator (GKA, PF-04991532) might also activate ChREBP (46). We found that administration of GKA alone increased G6pc expression 3-fold, and in conjunction with glucose gavage, further increased $G 6 p c$ expression along with ChREBP- $\beta$ and its transcriptional targets (Figure 3D). We tested ChREBP activation with 1 additional $\mathrm{CHO}$, glycerol, which is a preferred hepatic gluconeogenic substrate. Glycerol metabolites enter the hepatic glycolytic/gluconeogenic carbon pools at the triose-phosphate level, similar to fructose metabolites. Glycerol acutely and robustly stimulated expression of hepatic ChREBP- $\beta$ and its transcriptional targets including G6pc (Figure 3E). Thus, activation of hepatic ChREBP is not specific to fructose ingestion. Any carbohydrate that can increase the intrahepatic hexose- and triose-phosphate pool can likely activate hepatic ChREBP.

Figure 7. HGP is unchanged in chow-fed ChKO. (A) Glycemia, (B) serum insulin levels, and (C) HGP were measured after a 4-hour fast in 5-month-old live, conscious, chow-fed WT and ChKO mice ( $n=5$ per group). (D) Hepatic G6PC activity, (E) hepatic G6P levels, and (F) hepatic glycogen levels were measured in liver obtained from mice euthanized immediately after blood was obtained for the turnover measurement. $P$ values were obtained by Student's $t$ test. ${ }^{*} P<0.05$ compared with chow. Bars represent the mean \pm SEM.

ChREBP is necessary for fructose-induced hepatic gene expression and conversion of fructose to glucose. To examine whether fructose-induced gene expression in the liver depends on ChREBP, ChKO mice and their wild-type (WT) littermates were gavaged with water or fructose and changes in hepatic gene expression were examined 100 minutes later. Baseline levels of lipogenic and fructolytic enzymes and G6pc were reduced in ChKO mice and failed to increase with fructose gavage, demonstrating that ChREBP is required for basal and fructose-induced changes in hepatic gene expression (Figure 4A). We did not observe an increase in G6PC activity in association with the increased G6pc gene expression in WT mice in this short time frame (Figure 4B).

A

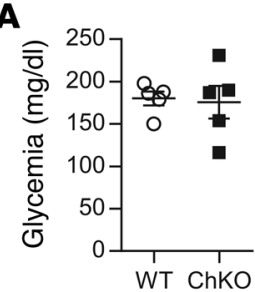

D

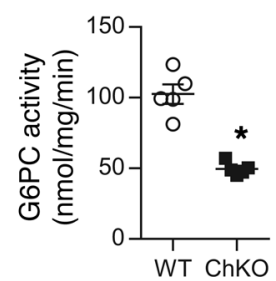

B

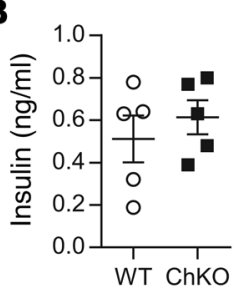

E

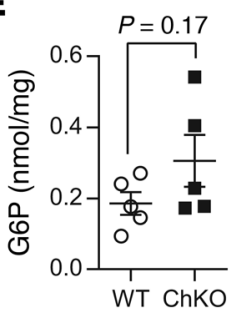

C

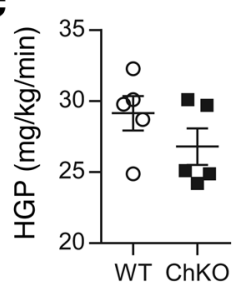

$\mathbf{F}$

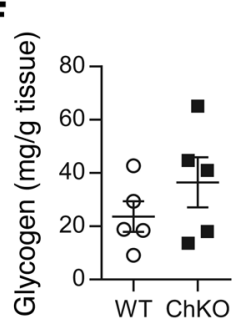



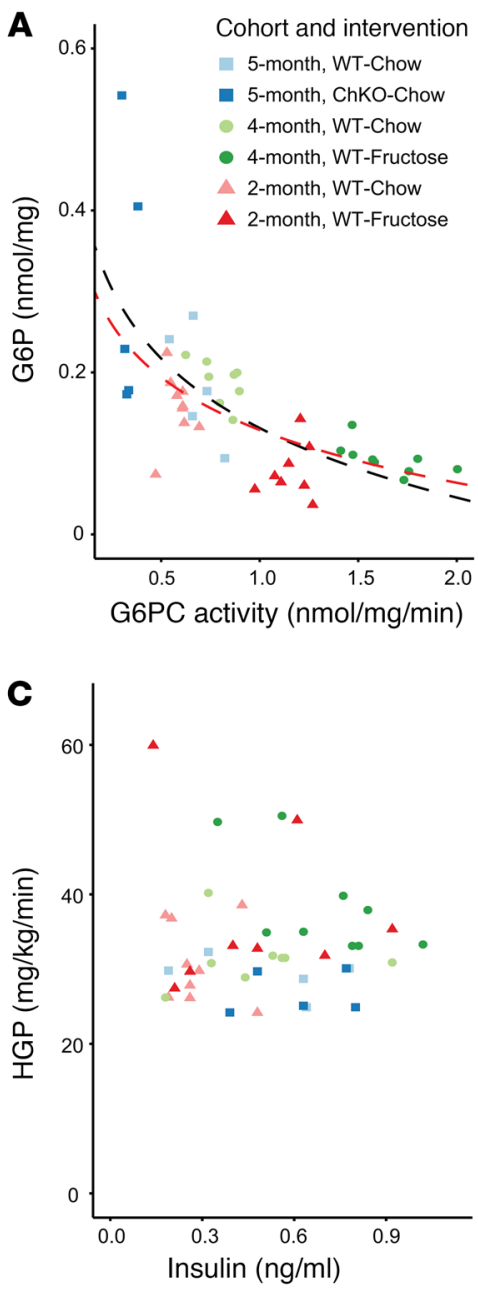
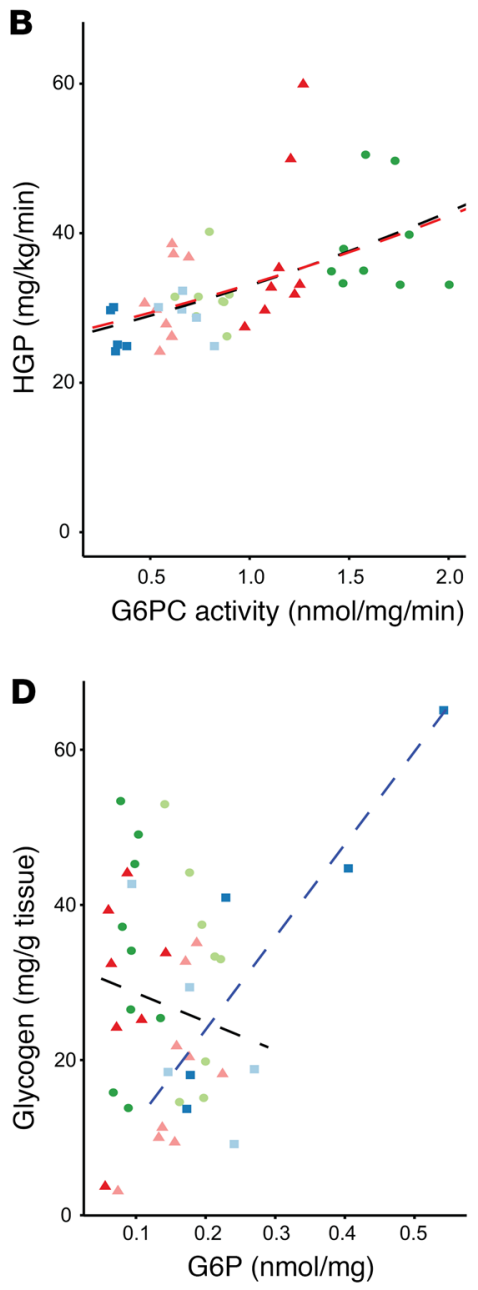

Figure 8. G6PC activity predicts hepatic G6P levels and HGP 4 hours after food removal. (A) Hepatic G6PC activity versus hepatic G6P levels ( $n=44)$, (B) hepatic G6PC activity versus $\operatorname{HGP}(n=44)$, (C) serum insulin levels versus HGP $(n=44)$, and (D) hepatic G6P levels versus hepatic glycogen $(n=43)$ in 3 mouse cohorts. Each data point represents an individual mouse. In $\mathbf{A}$ and $\mathbf{B}$, black dashed lines represent exponential fits including all mice. Red dashed lines represent exponential fits excluding ChKO mice. In $\mathbf{D}$, the black dashed line represents a linear fit excluding ChKO mice. The blue dashed line is the linear fit for ChKO mice only.

pyruvate in the media, demonstrating that fructose is readily used as a gluconeogenic substrate (Figure $5 \mathrm{~A}$ ). Feeding WT mice HFrD for 1 week increased glucose production from fructose by an additional $20 \%$, consistent with an adaptive response. In contrast, glucose production from fructose was reduced by $60 \%$ in $\mathrm{ChKO}$ mice (Figure 5B). Following fructose gavage, peripheral blood fructose clearance was impaired in $\mathrm{ChKO}$ mice (Figure 5C). Together, these results are consistent with a role for ChREBP in mediating an adaptive response to fructose ingestion and intrahepatic hexose-phosphate homeostasis (model, Figure 5D).

Hexose- and triose-phosphates are globally increased in ChKO mice. If ChREBP mediates hepatocyte $\mathrm{CHO}$ metabolite homeostasis, we reasoned that knocking out ChREBP should not only increase hepatic G6P levels, but might also globally increase hepatic hexose- and triose-phosphate levels. To assess this, we performed targeted metabolomics on livers harvested from ChKO mice and controls after water, fructose, or glycerol gavage. Consistent with our hypothesis, we observed that all measured glycolytic hexose- and triose-phos-

However, G6PC activity was 50\% reduced in both water- and fructose-treated ChKO mice compared with WT, consistent with the chronic reduction in G6pc gene expression in ChKO (Figure $4 \mathrm{~A})$. This reduction in G6PC is biochemically relevant, given the trend towards increased hepatic G6P levels in ChKO compared with WT with water gavage and a marked increase in G6P in ChKO following fructose gavage (Figure $4 \mathrm{C}$ ). This is consistent with previously reported increases in G6P levels in ChKO mice (22) and suggests a homeostatic model whereby increased hepatocellular hexose-phosphates, and possibly G6P itself (47), might activate ChREBP to enhance hexose- and triose-phosphate disposal. In addition to enhancing glycolytic flux and lipogenic flux (22), enhancing conversion of G6P to glucose through G6PC is another path by which activation of ChREBP might reduce intrahepatic hexose- and triose-phosphate levels as part of this homeostatic mechanism.

Based on this homeostatic model, we hypothesized that the ability of ChREBP to activate G6PC might be an important adaptive mechanism to mediate conversion of dietary fructose taken up in the liver first pass to glucose, which can then be readily utilized as fuel elsewhere in the body. To test this, we measured the conversion of fructose to glucose in isolated primary hepatocytes. Primary hepatocytes isolated from chow-fed WT mice generated 5 -fold more glucose from fructose compared with equimolar lactate plus phates as well as the pentose phosphate metabolite xyulose-5-phosphate, the fructolytic metabolite fructose-1-phosphate, and lactate tended to increase in ChKO livers (Supplemental Figure 2). Fructose gavage markedly increased fructose-1-phosphate levels in both ChKO mice and controls, and this did not occur following glycerol gavage (Supplemental Figure 3). Thus, increases in fructose-specific metabolites are not critical for activation of ChREBP. Fructose gavage tended to increase all of the measured hexoseand triose-phosphates and glycerol gavage tended to increase the majority of these same metabolites (Supplemental Figure 2). Overall, these results are consistent with a role for ChREBP in mediating hepatocyte hexose- and triose-phosphate homeostasis.

Fructose induces hepatic G6PC activity and glucose production in vivo. To determine whether high-fructose feeding can enhance glucose production in vivo, we measured glucose turnover in 4-month-old WT mice fed chow or HFrD for 2 weeks using isotopic dilution and obtained liver to measure hepatic G6P levels immediately after blood was obtained for the turnover measurement. Basal glycemia was not different between groups (Figure 6A). HFrD increased HGP (chow $31.48 \pm 1.41 \mathrm{mg} / \mathrm{kg} / \mathrm{min}, \mathrm{HFrD}$ $38.59 \pm 2.30 \mathrm{mg} / \mathrm{kg} / \mathrm{min}, P=0.02$, Figure $6 \mathrm{~B}$ ) and this occurred with a trend toward higher insulin levels (chow $0.48 \pm 0.08 \mathrm{ng}$ / $\mathrm{ml}, \mathrm{HFrD} 0.70 \pm 0.07 \mathrm{ng} / \mathrm{ml}, P=0.06$, Figure $6 \mathrm{C}$ ), consistent with insulin resistance. The increased HGP in HFrD was associated 
A

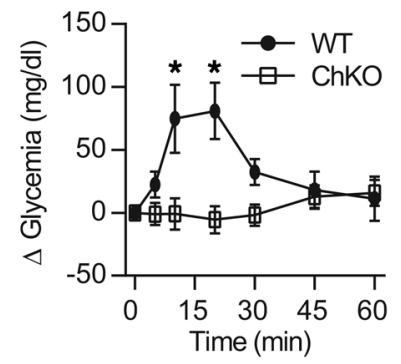

C

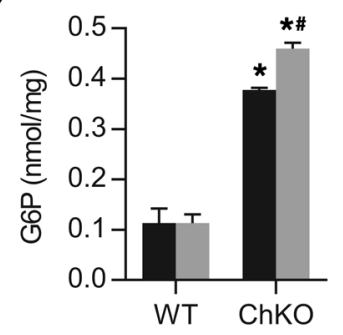

B

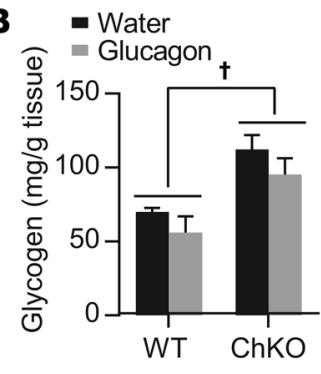

D

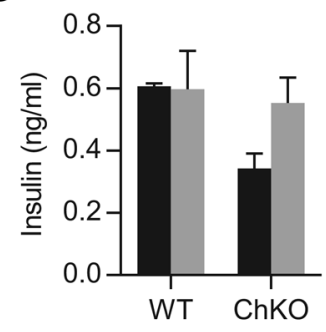

with increased G6PC activity (chow $118.7 \pm 5.3 \mathrm{nmol} / \mathrm{mg} / \mathrm{min}$, HFrD $243.9 \pm 9.9 \mathrm{nmol} / \mathrm{mg} / \mathrm{min}, P<0.001$, Figure $6 \mathrm{D})$. No differences in glycogen (Figure 6E) were observed.

Whereas fructose ingestion tends to acutely increase hepatic hexose-phosphate levels (Supplemental Figure 2), hepatic G6P levels were reduced 4 hours after food removal in mice fed HFrD for 2 weeks (chow $0.188 \pm 0.009 \mathrm{nmol} / \mathrm{mg}, \mathrm{HFrD} 0.093 \pm 0.006$ $\mathrm{nmol} / \mathrm{mg}, P<0.001$, Figure $6 \mathrm{~F})$. These results are consistent with a homeostatic model whereby increased G6PC activity resulting from increased $\mathrm{CHO}$ delivery to the liver in the fed state functions to reduce hepatic G6P levels and this is readily apparent after food is withdrawn. These results also indicate that increased G6PC activity within the physiological range is sufficient to drive glucose production, even in the face of lower levels of its immediate substrate, G6P. These results are consistent with the inference that genetic overexpression of G6PC may be sufficient to drive aberrant glucose production (15). Similar results were obtained in an independent cohort of 2-month-old mice.

We next assessed insulin signaling including the v-akt murine thymoma viral oncogene (AKT) (Figure 6G) and FOXO1A (Figure $6 \mathrm{H})$, both canonical factors in the insulin signaling pathway that regulate gluconeogenic gene expression (48). Phosphorylated AKT was highly variable in this cohort, but it was as high or higher in fructose-fed compared with chow-fed mice, consistent with intact insulin signaling. The abundance of cytosolic, insulin-inactivated FOXO1A (Figure 6H) was similar between diet groups and was nearly undetectable in nuclear fractions (data not shown), again consistent with intact insulin signaling. Thus, the increases in HGP and G6PC activity, and decreased G6P levels in highfructose-fed mice do not appear to be due to overt differences in hepatic insulin sensitivity.

We next asked whether ChREBP regulates glucose production in vivo. Because ChKO mice are intolerant to fructose, this was limited to chow-fed mice. Basal glycemia and insulin levels were not different between genotypes (Figure 7, A and B). There was a tendency for modestly lower HGP (Figure 7C) in ChKO, although this did not achieve statistical significance (WT $29.2 \pm 1.2 \mathrm{mg} / \mathrm{kg} / \mathrm{min}$,

Figure 9. ChREBP is essential for glucagon-stimulated glucose production. (A) Changes in blood glucose levels were measured after glucagon (20 $\mu \mathrm{g} / \mathrm{kg}$ body weight, ip) administration in ad libitum-fed 8- to 13-week-old male WT and ChKO mice ( $n=6-9$ per group) after 1 week on HDD. $P$ values were obtained by Student's $t$ test. ${ }^{*} P<0.05$ compared with ChKO at the indicated time point. After 2 weeks on $\mathrm{HDD}$, (B) hepatic glycogen levels, (C) hepatic G6P levels, and (D) serum insulin levels were measured 20 minutes after injection with either glucagon ( $20 \mu \mathrm{g} / \mathrm{kg}$ body weight) or water ( $n=3-5$ per group). $P$ values were obtained by 2 -way ANOVA. ${ }^{*} P<0.05$ compared with WT within treatment; ${ }^{*} P<0.05$ compared with water within genotype. ${ }^{\dagger} P<0.05$ main effect of genotype. Values are the mean $\pm \mathrm{SEM}$.

ChKO $26.8 \pm 1.3 \mathrm{mg} / \mathrm{kg} / \mathrm{min}, P=0.20$ ). ChKO mice again demonstrated a $50 \%$ reduction in $\mathrm{G} 6 \mathrm{PC}$ activity (Figure 7D) with a trend towards increased G6P and glycogen levels (Figure 7, $\mathrm{E}$ and F).

As glucose production is ultimately determined by the activity of G6PC and the immediate availability of its substrate, G6P, we sought to examine the molecular factors important for regulating glucose production in vivo. We performed a pooled analysis assessing the relationship between HGP, G6PC activity, G6P levels, and insulin across the cohorts described above (5-month-old, chowfed, ChKO, and littermate controls; 4-month-old, WT mice fed chow vs. HFrD for 2 weeks; and 2-month-old WT mice fed chow vs. $\mathrm{HFrD}$ for 2 weeks). In this large group of mice, G6P levels decayed exponentially with increasing G6PC activity (model: G6P $\sim \ln (\mathrm{G}-$ $6 \mathrm{PC}$ activity), $\mathrm{R}^{2}=0.47, P=3.06 \times 10^{-7}$, Figure $8 \mathrm{~A}$ and Supplemental Figure 4A). The exponential relationship between G6PC activity and G6P is similar when ChKO mice, which appear to define the G6P asymptote as G6PC activity declines, are removed from the analysis $\left(\mathrm{R}^{2}=0.43, P=6.17 \times 10^{-6}\right.$, Figure $8 \mathrm{~A}$ and Supplemental Figure $4 \mathrm{~B})$. Across this set of mice, HGP positively correlates with G6PC activity $\left(\mathrm{R}^{2}=0.34, P=3.68 \times 10^{-5}\right.$, Figure $8 \mathrm{~B}$ and Supplemental Figure 5), with a lower limit of $25.5 \mathrm{mg} / \mathrm{kg} / \mathrm{min}\left(P<2 \times 10^{-16}\right)$. Although G6P is the immediate substrate for glucose production, G6P levels inversely correlate with $\mathrm{HGP}\left(\mathrm{R}^{2}=015, P=0.009\right.$, Supplemental Figure 6, A and B). After accounting for the strong effect of G6PC activity on HGP, G6P levels have no independent correlation with HGP (Supplemental Figure 6C). Together, these results indicate that G6PC activity is a major determinant of glucose production and that within the physiological range tested here, G6PC activity is a more important factor than the immediate availability of substrate. The exponentially increasing G6P levels in both ChKO mice and chow-fed mice with lower levels of G6PC activity may, by mass action, compensate for reduced G6PC activity to increase HGP and help define the lower limit of HGP observed in Figure 8B. Similarly, the exponential decay in G6P levels with increasing G6PC activity may, by limiting substrate availability, define the upper limit of HGP. Importantly, ambient insulin levels did not correlate with HGP $\left(\mathrm{R}^{2}=0.001, P=0.82\right.$, Figure 8 C). Moreover, by multiple regression analysis, insulin levels had minimal impact on the relationship between G6PC activity and HGP (compare Supplemental Figures 5 and $7 \mathrm{~A}$ ). After accounting for the effect of G6PC activity on HGP, there is a tendency for insulin to negatively correlate with HGP, although this does not achieve statistical significance $\left(R^{2}=0.05, P=0.128\right.$, Supplemental Figure 7, $A$ and $B)$. Absent evidence of impaired insulin signaling resulting from 2 weeks on an HFrD (Figure 6), these results indicate that the natural variation in endogenous insulin levels among chow- and 
A

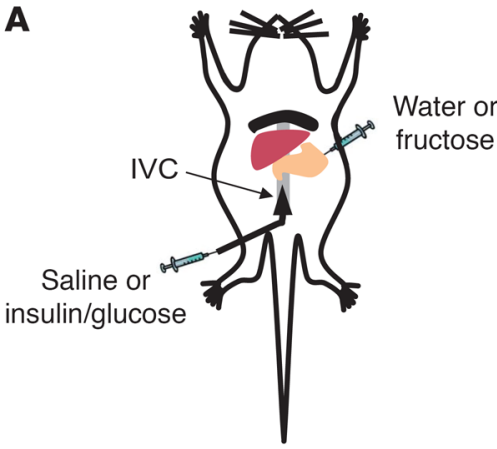

D

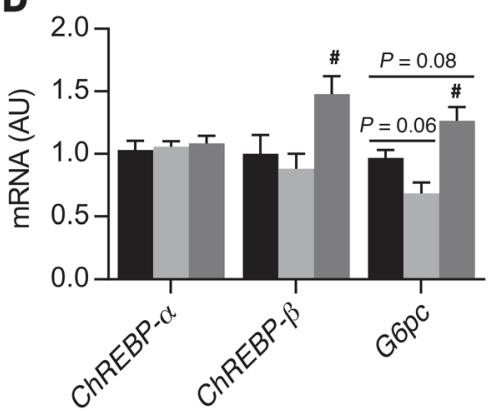

$\mathbf{F}$

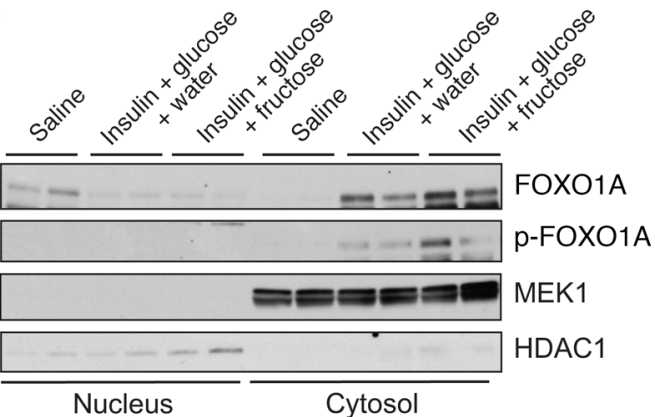

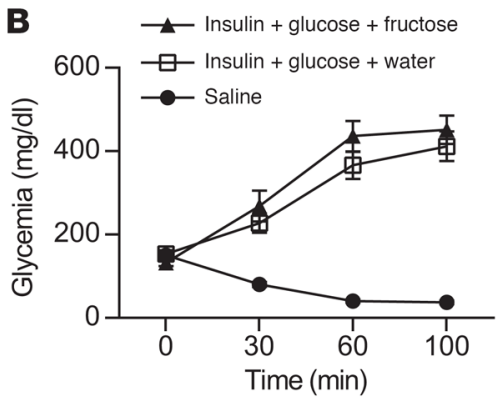
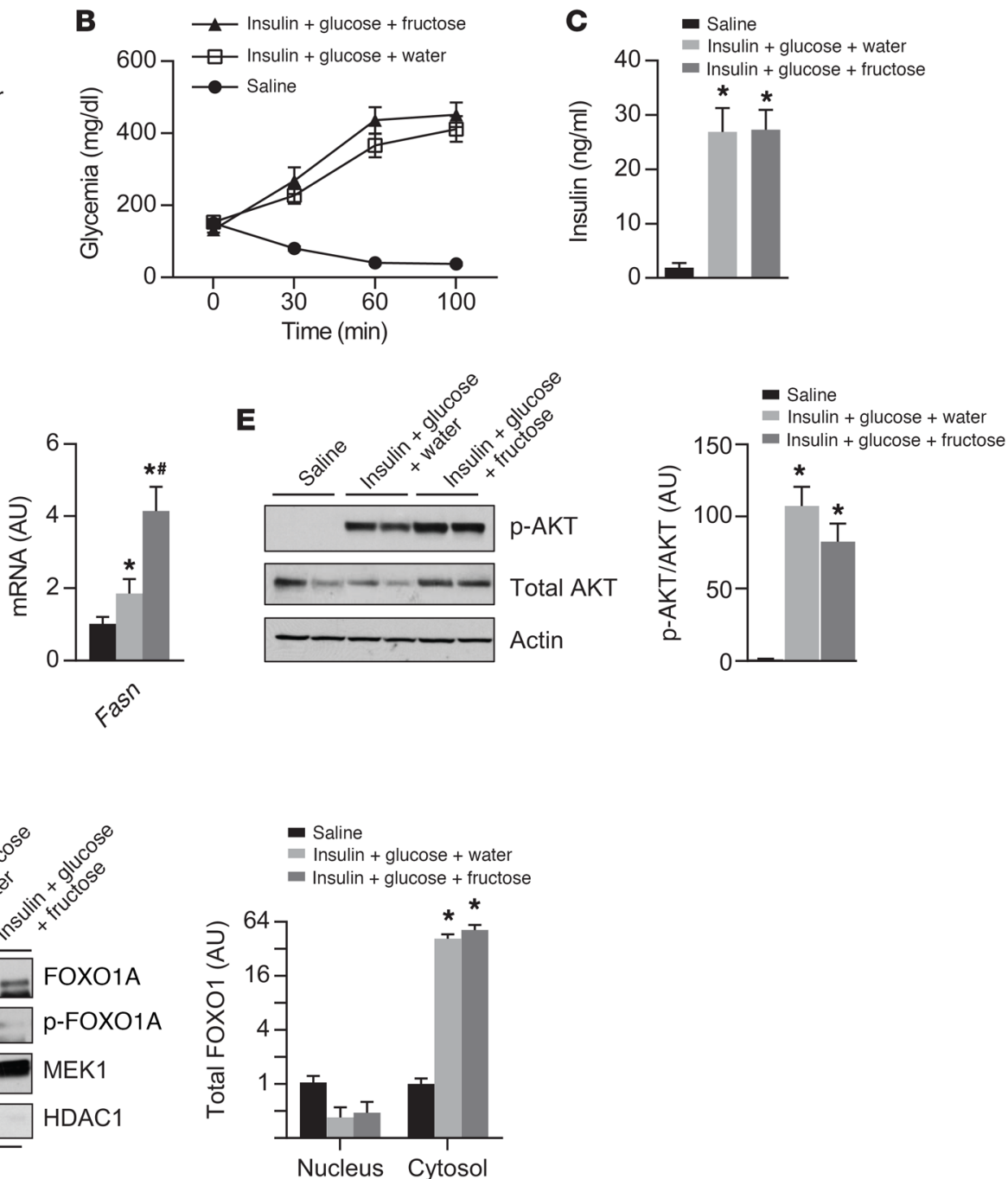

Figure 10. ChREBP upregulates G6pc despite activated hepatic insulin signaling. (A) Schematic diagram illustrating the experimental design. Insulin (1.5 $\mathrm{mU} / \mathrm{kg} / \mathrm{min}$ ) and glucose (20 mg/kg/min) were infused into 8-week-old C3H/He) mice after 5 hours of fasting and water or fructose (4 g/ kg) was injected into the stomach. Mice were sacrificed 100 minutes later. $P$ values were obtained by 1 -way ANOVA. ${ }^{*} P<0.05$ compared with saline; ${ }^{\#} P<0.05$ compared with insulin + glucose + water ( $n=11$ or 12 per group). (B) Glycemia was measured in tail vein blood throughout the duration of the experiment and (C) serum insulin was measured at termination. (D) Hepatic gene expression was measured by qPCR. (E) Representative Western blots of hepatic total AKT and phospho-AKT (Ser473), with quantification of the AKT/phospo-AKT ratio. (F) Nuclear and cytosolic F0X01A and phospho-F0X01A (Ser256) with quantification of total F0X01A normalized for the saline group within each cellular compartment ( $n=4$ per group). Values are the mean \pm SEM.

high-fructose-fed mice has minimal impact on the endogenous physiological mechanisms that govern the robust relationships between G6PC activity, hepatic G6P levels, and glucose production shortly after food removal.

As G6P is also an important allosteric activator of glycogen synthesis and inhibitor of glycogenolysis, we examined the relationship between G6P and glycogen in these cohorts. Among WT and control mice on either diet, we observed no significant correlation between hepatic G6P levels and glycogen levels $\left(R^{2}=0.02\right.$, $P=0.35$, Figure $8 \mathrm{D})$. In contrast, in ChKO mice, hepatic glycogen and G6P levels strongly correlate $\left(\mathrm{R}^{2}=0.85, P=0.02\right)$. This aberrant positive correlation may reflect inefficient flux of G6P to glucose due to reduced G6PC activity. This may result in aberrantly increased G6P levels, thus limiting glycogen breakdown. These results suggest that ChREBP through regulating the expression of metabolic enzymes including G6pc calibrates hepatocyte G6P levels, which may be important for coordinated regulation of glycogenolysis, glucose production, and other well-established terminal fates of hepatic hexose- and triose-phosphates such as lipogenesis.

Given the limitation in subjecting $\mathrm{ChKO}$ mice to diets containing fructose and the absence of marked changes in glucose production between chow-fed ChKO and controls, we sought another means to test the physiological significance of reduced G6PC activity in ChKO mice by testing the glycemic response to glucagon. Glucagon robustly increased glycemia in WT, but not ChKO mice (Figure 9A). We next examined the effects of glucagon on hepatic glycogen and G6P levels. As expected, glycogen levels were higher in ChKO mice (Figure 9B). Glucagon did not increase hepatic G6P levels in WT mice, as G6P hydrolyzed from glycogen was likely efficiently converted to glucose and other end fates via 
A

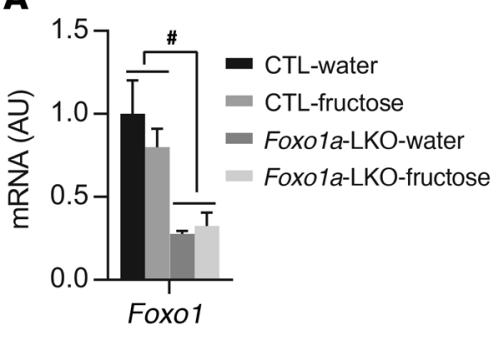

E

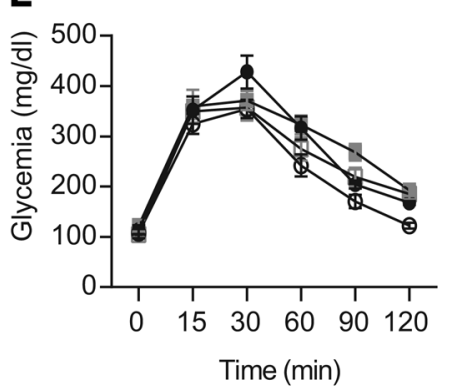

B

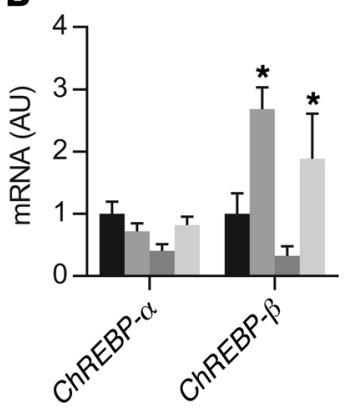

$\mathbf{F}$

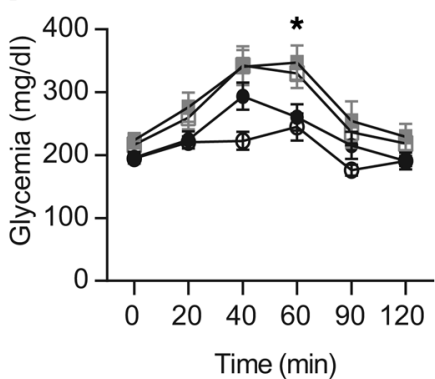

C
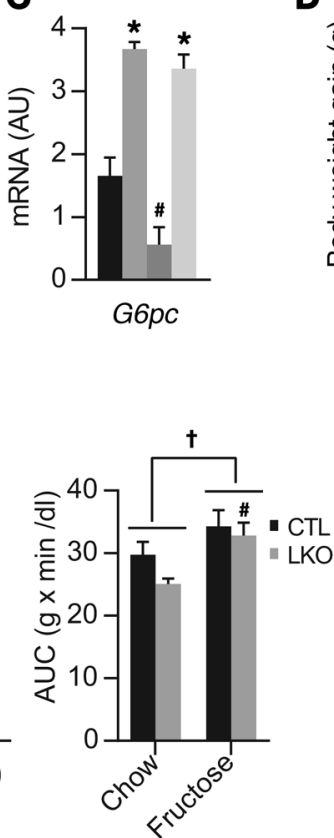

D

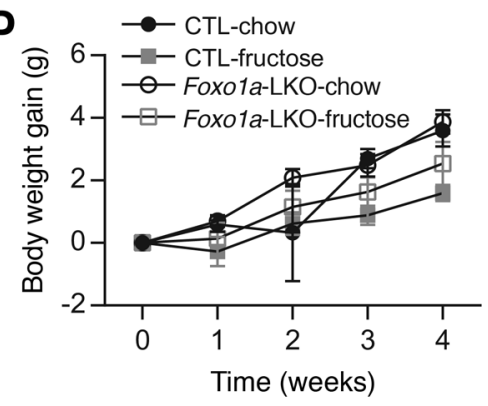

G

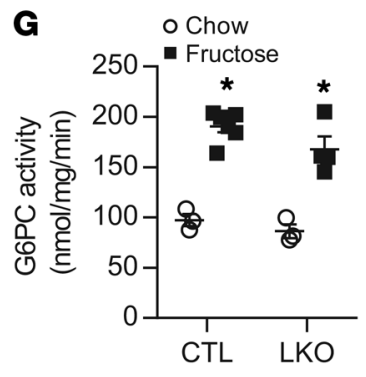

Figure 11. Fructose activates ChREBP and induces G6PC and glycerol intolerance independently of F0X01A. Five-hour-fasted, 8- to 10-week-old CTL and Foxo1a-LKO mice were gavaged with water or fructose ( $4 \mathrm{~g} / \mathrm{kg}$ body weight) and sacrificed 100 minutes later. (A-C) Hepatic gene expression was measured by qPCR ( $n=3$ or 4 per group). ${ }^{*} P<0.05$ compared with water; ${ }^{\sharp} P<0.05$ compared with WT. Values are the mean \pm SEM. (D) Weight gain of male CTL and Foxo1a-LKO mice fed chow versus HFrD for 4 weeks $\left(n=6-8 /\right.$ group). (E) Glucose tolerance test and (F) glycerol tolerance test. ${ }^{*} P<0.05$ for chow vs. HFrD within genotype with area under the curve (AUC) for this cohort. ${ }^{t} P<0.05$ main effect of HFrD; $\#<0.05$ vs. chow-LKO. (G) Hepatic G6PC activity from ad libitum-fed mice. $n=3$-6/group, ${ }^{*} P<0.05$ within genotype versus chow. Values and bars are means \pm SEM. All $P$ values were obtained by 2-way ANOVA.

glycolysis (Figure 9, A and C). G6P levels were markedly elevated in ChKO mice compared with WT, and glucagon further increased G6P levels in ChKO (Figure 9C). Thus, glucagon can stimulate glycogenolysis in ChKO mice, but, in contrast with WT mice, G6P produced from glycogenolysis is trapped within the glycolytic/ gluconeogenic carbon pool. Consistent with the results from the turnover experiments, we observed no correlation between G6P and glycogen levels in WT mice (Supplemental Figure 8, A and B). However, within the ChKO group, by multiple regression, G6P levels correlate with glycogen levels independently of glucagon treatment $(P=0.018$, Supplemental Figure $8 C)$. No differences were observed in serum insulin levels between groups (Figure 9D). These results support an important role for ChREBP to coordinate hepatic G6PC activity to regulate HGP and hepatic hexose-phosphate homeostasis.

ChREBP regulates G6pc independently of insulin signaling. To examine whether sugar-induced ChREBP-mediated transactivation of G6pc might contribute to an apparent increase in hepatic insulin resistance, we tested whether the effect of fructose to enhance hepatic G6pc expression can overcome the effect of insulin to suppress it. In anesthetized mice, we infused insulin (1.5 $\mathrm{mU} / \mathrm{kg} / \mathrm{min})$ and glucose $(25 \mathrm{mg} / \mathrm{kg} / \mathrm{min})$ through the inferior vena cava (IVC) and injected water or fructose into the stomach (Figure 10A). We achieved marked hyperinsulinemia and hyperglycemia in mice infused with glucose and insulin throughout the duration of the experiment (Figure 10, B and C). Consistent with our glucose gavage data, hyperglycemia generated via glucose and insulin infusion did not increase hepatic ChREBP- $\beta$ expression (Figure 10D). Expression of ChREBP- $\beta$ increased $50 \%$ in glucose- and insulin-infused mice when provided fructose (Figure 10D). The insulin/glucose infusion tended to suppress hepatic G6pc expression. However, G6pc expression was increased in insulin- and glucose-infused mice following fructose treatment, suggesting that the effect of fructose to increase G6pc expression is dominant over the effect of a combination of insulin and glucose to suppress it (Figure 10D). As expected, the combined insulin and glucose infusion increased Fasn expression, and this was further enhanced with fructose administration (Figure 10D). The effect of fructose to increase G6pc expression in the setting of hyperglycemia and hyperinsulinemia occurs in the setting of intact insulin signaling as evidenced by robust phosphorylation of AKT (Figure 10E) and FOXO1A (Figure 10F). Together, these results indicate that ChREBP can transactivate expression of G6pc even when hyperinsulinemia excludes FOXO1A from the nucleus.

FOXO1A positively regulates gluconeogenic enzyme expression including G6pc (48), and insulin-mediated inhibition of FOXO1A accounts for the majority of insulin-mediated suppression of the expression of $G 6 p c$ and other gluconeogenic enzymes (49, 50). We sought to confirm that fructose- and ChREBP-mediated transactivation of G6pc is independent of FOXO1A. To do so, we tested the ability of fructose to induce G6pc in liver-specific Foxo1a knockout mice (Foxola-LKO) versus control littermates (CTL). Foxo1a expression was reduced by 70\% in Foxo1a-LKO (Figure 11A). Fructose gavage increased ChREBP- $\beta$ expression similarly in both 
A
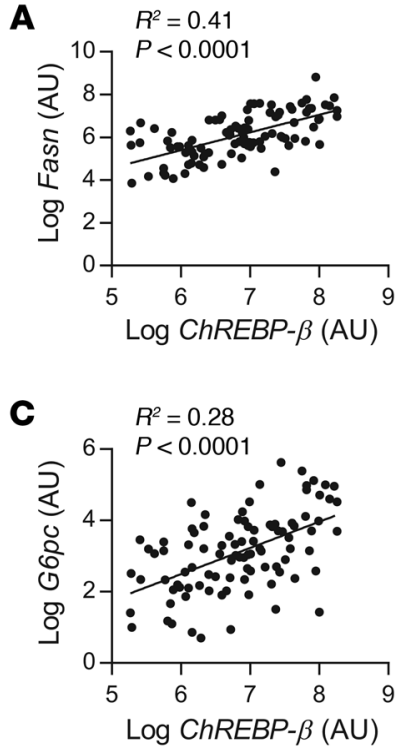
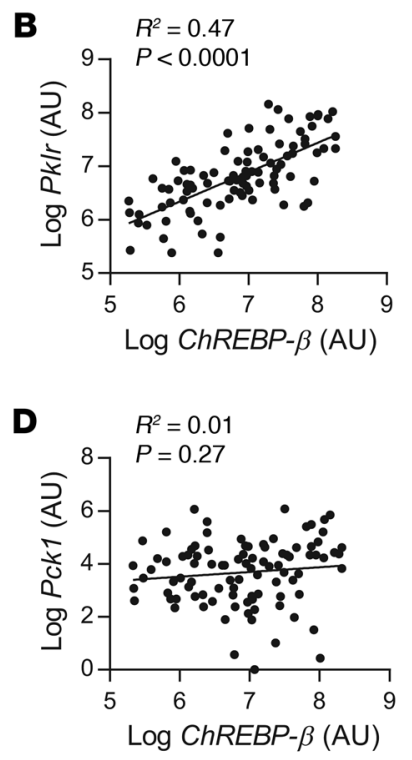

CTL and Foxo1a-LKO (Figure 11B). G6pc expression was reduced in water-gavaged Foxo1a-LKO compared with CTL, consistent with FOXO1A's known role in regulating G6pc expression (Figure 11C). Fructose gavage produced similar increases in G6pc expression in both CTL and Foxo1a-LKO, demonstrating that fructose-mediated regulation of $G 6 p c$ is independent of the insulin-regulated factor FOXO1A (Figure 11C). No significant differences were noted between genotypes in serum glucose, triglyceride, or insulin following water or fructose gavage (Supplemental Figure 9).

We next challenged CTL and Foxo1a-LKO mice with chow versus HFrD for 4 weeks. In this time frame, weight gain was similar across genotypes and diets (Figure 11D) and glucose intolerance did not develop (Figure 11E). Nevertheless, 4 weeks of HFrD was sufficient to enhance glucose excursion following glycerol administration in CTL and Foxo1a-LKO (Figure 11F). G6PC activity was increased 2-fold in both CTL and Foxo1a-LKO (Figure 11G). Thus, fructose-mediated activation of ChREBP stimulates expression of lipogenic enzymes synergistically with hepatic insulin signaling and stimulates enzymes essential for glucose production independently of insulin signaling, providing a potentially novel mechanism contributing to the apparent paradox of hepatic insulin resistance.

The ChREBP-G6PC signaling axis is conserved in humans. To determine whether a ChREBP-G6PC axis might also participate in regulating glucose production in humans, we examined expression of ChREBP and its transcriptional targets in human liver samples. Expression of $C h R E B P-\beta$ positively correlated with canonical ChREBP targets including Pklr and Fasn and also strongly correlated with G6pc (Figure 12, A-C). No correlation was observed between the expression of ChREBP- $\beta$ and Pck1, a gluconeogenic enzyme that is not regulated by ChREBP (Figure 12D). It should be noted that the expression of ChREBP- $\alpha$ also correlated with the expression of ChREBP target genes but less so than ChREBP- $\beta$ (Supplemental Figure 10). By multiple regression analysis $C h R E B P-\beta$, but not ChREBP- $\alpha$ expression correlates with the canonical ChREBP targets Fasn and Pklr (Supplemental Figure 11). These results are consistent with our prior data indicating that ChREBP- $\beta$ expression best reflects total ChREBP activity (36). By

Figure 12. A ChREBP-G6PC signaling axis is conserved in human liver. The correlation between ChREBP- $\beta$ gene expression and (A) Fasn, (B) Pklr, (C) G6pc, and (D) Pck1 were compared in liver biopsy samples from overnight-fasted human subjects with NAFLD. Each data point represents an individual person $(n=95)$. $P$ values were obtained by linear regression.

multiple regression analysis, the correlations between ChREBP- $\alpha$ and ChREBP- $\beta$ with $G 6 p c$ are independent of each other and the correlation between $C h R E B P-\beta$ with $G 6 p c$ is much stronger than that of ChREBP- $\alpha$. These data suggest that a distinct gene expression program may exist that coregulates $P c k 1, G 6 p c$, and ChREBP- $\alpha$ expression. Altogether, these data are consistent with the conclusion that ChREBP may contribute to lipogenic gene expression and apparent hepatic insulin resistance at the level of G6pc expression in humans as it does in mice.

\section{Discussion}

Here we demonstrate that fructose ingestion, but not glucose ingestion, robustly and acutely activates the key metabolic transcription factor ChREBP in the livers of mice. This is associated with increased expression of genes encoding enzymes involved in glycolysis, fructolysis, DNL, as well as G6PC, the final step in HGP. ChREBP is required for fructose-induced changes in these metabolic programs, as this is absent in ChKO mice. We show that ChREBP is also important for efficient conversion of fructose to glucose in hepatocytes and whole-body fructose clearance, which suggests that this molecular mechanism may have evolved to sense dietary sugar consumption in order to enhance its efficient metabolism.

When consumed in excess, fructose causes many features of metabolic disease. In this study, mice fed either HDD or HFrD developed obesity and hepatic steatosis, but only those fed an HFrD developed hyperinsulinemia, hypertriglyceridemia, and impaired glucose and glycerol tolerance, indicating that fructose has a specific deleterious effect on glucose homeostasis and hyperlipidemia independently of fatty liver. This is consistent with data showing that excessive fructose, but not glucose consumption, causes hypertriglyceridemia and impaired glucose metabolism in overweight human subjects (29). Fructose-induced alterations in lipid metabolism are likely in part due to activation of ChREBP and its lipogenic gene program, consistent with data showing that polymorphisms in the ChREBP locus are associated with circulating triglycerides in human populations $(51,52)$. How activating ChREBP might participate in impaired glucose homeostasis is less obvious. We show that ChREBP under the influence of fructose ingestion directly transactivates $G 6 p c$ expression, which is one mechanism by which activating ChREBP might contribute to impaired glucose homeostasis. This is supported by data showing that overexpression of G6PC is sufficient to cause hyperinsulinemia and impair glucose tolerance in rodents (15).

ChREBP was discovered based upon observations that glucose could stimulate gene expression in primary hepatocyte culture independently of insulin (53). In contrast, in vivo, orally administered fructose, but not glucose robustly activates hepatic ChREBP. Moreover, several hours of hyperglycemia resulting from glucose infusion did not activate hepatic ChREBP in vivo. We have observed marked alterations in the expression of ChREBP, fructolytic enzymes, and other key metabolic enzymes occurring 
during hepatocyte isolation that persist in primary hepatocyte culture, including upregulation of hexokinases not highly expressed in liver in vivo (data not shown). We suspect that these changes contribute to the reported ability of glucose to activate hepatic ChREBP in primary culture, in contrast with our results in vivo.

The ability of fructose, but not glucose, to acutely activate ChREBP in vivo is likely due to differences in how the intact liver metabolizes these 2 simple sugars. The majority of an oral glucose load escapes first-pass hepatic metabolism due in part to sequestration of GCK in an inactivated state by $\operatorname{GCKR}(39,54)$. In contrast, fructose is largely extracted by the liver first pass (26). Moreover, the fructose metabolite fructose-1-phosphate produced by ketohexokinase destabilizes the interaction between GCK and GCKR, permitting hepatocellular glycolytic flux (55). Thus, fructose may activate ChREBP either directly through metabolites derived from fructose or indirectly by activating hepatic GCK and enhancing glycolytic flux, which might then activate ChREBP. Our results show that glucose plus a liver-specific GKA can also robustly activate hepatic ChREBP, which indicates that a fructose-specific metabolite is not required for ChREBP activation. The fact that glycerol, which is also rapidly phosphorylated in the liver, can also acutely activate ChREBP demonstrates that multiple substrates that can enter the glycolytic/gluconeogenic carbon pool are sufficient for ChREBP activation and that activation of GCK is not a requirement. The specific metabolite and mechanism by which ChREBP is activated remains uncertain (56).

Our results are consistent with the hypothesis that hepatic ChREBP serves as a sensor for dietary sugar ingestion. Dietary sugar occurs predominantly in the form of sucrose -1 glucose monosaccharide joined to 1 fructose monosaccharide. An evolved role for hepatic ChREBP in the metabolic response to dietary sugar would help explain the counterintuitive observation that activation of ChREBP increases both the expression of glycolytic enzymes and $G 6 p c$, the final step in glucose production. Sucrose-induced activation of ChREBP and G6PC would be important to enhance storage of ingested glucose in the liver as glycogen while simultaneously converting ingested fructose into glucose for use elsewhere in the body. This is supported by our findings here. This is also consistent with the fact that fructose ingestion, through hepatic ChREBP activation, stimulates expression of some gluconeogenic enzymes (e.g., G6pc) but not others (e.g., Pck1). Fructose metabolites enter glycolytic/gluconeogenic carbon pools at the triose-phosphate level, distal to Pck1 in the gluconeogenic pathway. Thus, efficient conversion of fructose to glucose requires G6PC activity, but not PCK1 activity. Lastly, the fact that other substrates such as glycerol or the combination of glucose and a GKA stimulate expression of fructolytic enzymes along with ChREBP, when no fructose has been provided, is consistent with the notion that ChREBP is a major effector of the response to dietary fructose.

From an organismal perspective, hepatic ChREBP plays a role in dietary sugar metabolism. From a hepatocyte-centric perspective, we and others have hypothesized that ChREBP might function to regulate hepatocyte triose- and hexose-phosphate homeostasis (17), and our results support this model.

Can ChREBP-mediated regulation of G6pc contribute to the hepatic insulin resistance paradox in obese, insulin-resistant subjects? More specifically, can carbohydrate-activated ChREBP induce $G 6 p c$ and overcome insulin's ability to suppress it? These questions are motivated by reports that ChREBP- $\beta$ expression as a marker of ChREBP activity is increased in livers from obese, insulin-resistant humans $(57,58)$. Here we show that fructose feeding induces G6PC activity and HGP and this is dominant over insulin's effect of reducing $G 6 p c$ expression via regulation of FOXO1A. After food withdrawal, the fructose-mediated increase in G6PC reduces G6P levels to close the hexose-phosphateChREBP-G6pc homeostatic loop.

Across a large cohort of chow- versus fructose-fed mice, the strong relationships between G6PC activity, HGP, and G6P levels are insulin independent. Although exogenous insulin infusions can readily regulate glucose production by regulating glycogenolysis, G6P levels, and other mechanisms, the natural variation in endogenous insulin levels appears to have little impact on the mechanisms regulating variation in glucose production in the paradigm studied here. It will be important in the future to extend this analysis to determine whether this conclusion can be generalized to other nutritional and genetic contexts affecting HGP.

Our studies have focused on gluconeogenic enzyme expression and basal glucose production and do not exclude the possibility that selective defects in hepatic insulin signaling also contribute to the pathogenic paradox of hepatic insulin resistance by regulating HGP in other contexts. In fact, insulin action in the liver can acutely reduce glucose production $(10,11)$, and this occurs in a time frame where gluconeogenic enzyme expression is largely unaffected. Thus, additional mechanisms are required to explain resistance to insulin's effect of acutely reducing glucose production, as is often observed in euglycemic-hyperinsulinemic clamp procedures.

Our results potentially provide a new perspective on this important phenomenon. We show that hepatic G6PC activity positively correlates with HGP and strongly and inversely correlates with hepatic G6P levels. This shows that increased G6PC activity can overcome a relative deficit in its immediate substrate to increase HGP. The major mechanism by which insulin is thought to acutely reduce HGP is by inhibiting net glycogen breakdown in the liver and reducing G6P availability as substrate for G6PC (reviewed in ref. 59). However, the effect of insulin to inhibit glycogen phosphorylase and stimulate glycogen synthase is strongly countered by the ability of low G6P levels to stimulate glycogen breakdown and limit glycogen synthesis. In fact, recent evidence indicates that the allosteric effect of G6P on glycogen synthesis and breakdown are dominant over insulin's effects (60). Thus, low hepatic G6P levels caused by high G6PC activity might limit insulin's ability to reduce glycogen breakdown, further reduce G6P levels, and limit HGP. It remains possible that in settings where hepatic glycogen stores are depleted, insulin may be able to acutely reduce glucose production via its peripheral effects to suppress adipose lipolysis and delivery of substrate to the liver $(8,9)$. Additional studies will be required to investigate these hypotheses.

Lastly, we show that in human liver biopsy samples, ChREBP- $\beta$ expression strongly correlates with $G 6 p c$ expression as well as the expression of DNL enzymes, indicating that this is a major mode of $G 6 p c$ regulation in humans in vivo. Thus, this mechanism likely contributes to the apparent paradox of selective hepatic insulin resistance in humans as it does in mice. 


\section{Methods}

Materials. Glucose, fructose, G6P, G6P dehydrogenase (G6PD), resazurin, diaphorase, flavin mononucleotide (FMN), glycogen type III, amyloglucosidase, and perchloric acid were purchased from Sigma-Aldrich. Nicotinamide adenine dinucleotide (NAD) was purchased from Roche. Insulin was purchased from Eli Lilly. Glucagon was purchased from Bedford Laboratories. Glucose oxidase reagent was purchased from Thermo Scientific. Insulin ELISAs were purchased from Crystal Chem (Ultra Sensitive Mouse Insulin ELISA) or EMD Millipore (3P Linco Insulin ELISA Kit, EZRMI13K). The GKA PF-04991532 was provided by J. A. Pfefferkorn (Pfizer). All metabolite standards for mass spectrometry were purchased from Sigma-Aldrich.

Human liver samples. The subjects for this study were a subgroup of patients enrolled in an NAFLD registry at Beth Israel Deaconess Medical Center (BIDMC) beginning in 2009, which is a prospective study that enrolls subjects with biopsy-proven NAFLD. Liver biopsy specimens were stored at $-80^{\circ} \mathrm{C}$.

Animals and diets. Both male and female mice were used at ages as indicated in specific experiments. ChKO mice, described previously (22), were obtained from The Jackson Laboratory (stock 010537). ChKO mice were backcrossed onto a $\mathrm{C} 3 \mathrm{H} / \mathrm{HeJ}$ background (Jackson Laboratory, stock 000659) for more than 7 generations. Foxo1a floxed mice were provided by W.C. Aird (BIDMC) and have been previously described (61). Liver-specific Foxo1a knockout mice and controls were generated by crossing Foxo1 a floxed heterozygous mice on a mixed $\mathrm{C} 57 \mathrm{BL} / 6 \mathrm{~J}(50 \%)$ and $\mathrm{C} 3 \mathrm{H} / \mathrm{HeJ}(50 \%)$ background with albumin-Cre (Jackson Laboratory stock 003574)-Foxo1a floxed heterozygotes also on a mixed C57BL/6J (50\%) and C3H/HeJ (50\%) background. Mice were housed and bred in a temperature-controlled animal facility with a 12-hour light/12-hour dark cycle and free access to food and water, at BIDMC. Before initiation of diet studies, the mice were maintained on a standard laboratory chow diet (Purina LabDiet 5008). Both the 60\% fructose (TD.89247, Harlan Teklad) and $60 \%$ dextrose (TD.05256) diets contained 20.2\% protein, $12.9 \%$ fat, and $66.8 \%$ carbohydrate.

Body weight and metabolic testing. Body weight was measured on a weekly basis. Analyses were performed using blood samples drawn from overnight fasted or fasted-refed (3 hours) mice. Blood was collected from the tail vein except in experiments where mice were anesthetized in order to freeze-clamp the liver. In such experiments, blood was collected from the IVC. Blood glucose was measured using a glucometer. Plasma triglyceride was measured using a colorimetric assay kit (Stanbio). For glucose or glycerol tolerance tests, mice were fasted for 5 hours and glucose $(1 \mathrm{~g} / \mathrm{kg}$ of body weight) or glycerol (1.5 g/ $\mathrm{kg}$ body weight) was administered via intraperitoneal (i.p.) injection.

Biochemical and enzymatic assays. Liver metabolites were extracted from mouse liver snap frozen using a freeze clamp under isoflurane anesthesia. Metabolites were extracted from the liver using perchloric acid (62). G6P fluorometric assays were performed as previously described (63). Neutral lipids were extracted from the liver based on the Folch method (64). A portion of the triglyceride-containing chloroform phase was collected, evaporated, and resuspended in a 2:1 mixture of butanol and Triton X-100/methanol (3:1). Triglyceride was then measured using a triglyceride kit purchased from StanBio according to the manufacturer's protocol and normalized to liver weight. G6PC activity assay was performed as previously described (65).
Immunoblotting. To generate nuclear versus cytosolic lysates, liver tissue was initially homogenized with $10 \mathrm{mM}$ Hepes (pH 7.9), $10 \mathrm{mM}$ $\mathrm{KCl}, 0.1 \mathrm{mM}$ EDTA, $0.1 \mathrm{mM}$ EGTA, $1 \mathrm{mM}$ DTT, $50 \mu \mathrm{g} / \mathrm{ml}$ digitonin, $0.5 \mathrm{mM}$ PMSF, and protease inhibitor cocktail (Sigma-Aldrich, catalog P8340). Nuclei were then separated by $1,000 \mathrm{~g}$ centrifugation for 10 minutes, followed by lysis in RIPA buffer containing $50 \mathrm{mM}$ Tris $(\mathrm{pH}$ 7.4), $150 \mathrm{mM} \mathrm{NaCl}, 1 \%$ (w/w) NP-40, 0.1\% (w/v) SDS, $1 \%$ (w/v) Na deoxycholate, $2 \mathrm{mM}$ EDTA, $50 \mathrm{mM} \mathrm{NaF}$, and $10 \mathrm{mM}$ NaPPi with $1 \mathrm{mM}$ PMSF and protease inhibitor cocktail. Nuclear and cytosolic lysates were then subjected to immunoblotting with the indicated antibodies: FoxO1 (Cell Signaling, catalog 2880), phospho-FoxO1 (Cell Signaling, catalog 9461), HDAC1 (EMD Millipore, catalog 06-720), phospho-Akt (Cell Signaling, catalog 4060), Akt (Santa Cruz, catalog sc-1618 and Cell Signaling, catalog 2920), MEK1 (Cell Signaling, catalog 8727), actin (Santa Cruz, catalog sc-1616), ChREBP (Novus Biologicals, cata$\log$ NB400-135), and HDAC2 (Cell Signaling, catalog 2540).

ChIP. ChIP was performed as previously described (66).

Primary hepatocyte culture and glucose production assay. Primary hepatocytes were isolated from 8- to 10-week-old WT and ChKO mice or 8- to 10-week-old C3H/HeJ mice fed either chow or HFrD for 1 week by perfusion with Liver Digest Medium (Invitrogen) followed by $100 \mathrm{~mm}$ mesh filtration and Percoll gradient centrifugation. Isolated cells were seeded into 24 -well plates at a density of $2 \times 10^{5}$ cells per well in William's E medium (Invitrogen) containing 10\% FBS and $2 \mathrm{mM}$ glutamine. Four hours after seeding, the medium was replaced with $0.5 \mathrm{ml}$ of glucose production buffer consisting of glucose-free DMEM without phenol red, supplemented with $5 \mathrm{mM}$ sodium lactate and $0.5 \mathrm{mM}$ sodium pyruvate or $5 \mathrm{mM}$ fructose. After 3 hours of incubation, the medium was collected and the glucose concentration was measured with a colorimetric glucose assay kit (Thermo Scientific). The readings were then normalized to the total protein content determined from whole-cell lysates.

Basal glucose turnover measurements. In vivo assessment of basal glucose turnover was performed by the National Mouse Metabolic Phenotyping Center at UMass Medical School in male, age-matched WT C3H/HeJ mice fed chow or HFrD for 2 weeks or male age-matched ChREBP KO mice and WT littermates fed chow diet following jugular vein cannulation surgery and recovery. Whole-body glucose turnover was assessed as previously described in 2-hour-fasted mice and after a 2-hour equilibration period (4 hours fasted) (67). Immediately following blood collection, mice were anesthetized, and liver was snap frozen in liquid nitrogen for further analysis.

Insulin-glucose infusion. Eight-week-old $\mathrm{C} 3 \mathrm{H} / \mathrm{HeJ}$ mice were fasted for 5 hours and then anesthetized using isoflurane. Catheters were inserted into the inferior vena cava. Mice were infused with insulin (50 mU/ kg priming dose followed by $1.5 \mathrm{mU} / \mathrm{kg} / \mathrm{min}$ constant infusion) and glucose $(25 \mathrm{mg} / \mathrm{kg} / \mathrm{min}$ constant infusion) for 100 minutes. Mouse liver was snap frozen in situ using a Wollenberg clamp.

qPCR. TRI reagent (MRC, catalog TR118) was used for RNA isolation from mouse liver and adipose tissue. RNA was reverse transcribed using a SuperScript VILO kit (Invitrogen). Gene expression was analyzed with the ABI Prism sequence detection system (SYBR Green; Applied Biosystems). Gene-specific primers were synthesized by IDT (see Supplemental Table 1). Each sample was run in duplicate, and normalized to RplpO RNA.

LC-MS for liver metabolites. The liquid chromatography-mass spectrometry (LC-MS) method for measurement of liver tissue metabolites was adapted from Buescher et al. (68). 
Additional details regarding the G6PC activity assay, ChIP experiments, liver metabolite LC-MS, and LC-MS for fructose clearance are described in Supplemental Methods.

Statistics. Results are presented as the mean \pm SEM. Differences between groups were examined for statistical significance by either 2-tailed Student's $t$ test, 1-way ANOVA followed by Tukey's post-hoc test, or 2-way ANOVA followed by Sidak's post-hoc test as appropriate in GraphPad Prism. A P value of less than 0.05 was considered significant. Statistical comparisons were performed on log-normalized data when variances between groups were not normally distributed. Metabolomics data were analyzed and heat maps were generated with $\mathrm{R}$ version 3.2.1. Multiple regression analysis of HGP data and graphs were generated with $\mathrm{R}$ version 3.2.4. Multiple regression analysis of human qPCR data was performed with SPSS release 18.0.0.

Study approval. Use of human liver samples was approved by the BIDMC institutional research board. All mouse studies were approved by the BIDMC Institutional Animal Care and Research Advisory Committee.

\section{Author contributions}

MK, SAK, and MAH conceived of, designed, performed, and interpreted mouse experiments and made figures. $\mathrm{LD}$ and $\mathrm{AF}$ assisted with designing, performing, and interpreting mouse experiments. JXW and SAT performed and interpreted fructose clearance MS measurements. HLN, HJK, and JKK designed and interpreted glucose turnover experiments, which were performed by HLN and
HJK. JKM and MB performed and interpreted metabolomics measurements and LD analyzed these data. ML provided human liver biopsy samples. MAH conceived of, designed, and supervised the experimental plan, interpreted experiments, and wrote the manuscript. MK, JKK, JKM, and MB edited the manuscript.

\section{Acknowledgments}

We thank John Riley, Greg McElroy, Jason Kong, and David Kim for their technical assistance. We thank Lauren Janes for assistance with Foxo1 a floxed mice. We thank Greg Tesz and Inna Astapova for helpful discussions. This work is supported by NIH grants P3ODK057521 and R01DK100425 (to MAH), T32DK007516 (to MK), and K23DK083439 (to ML). Part of this study was performed at the National Mouse Metabolic Phenotyping Center at UMass funded by NIH grant UC2 DK093000 (to JKK). We thank the Animal Metabolic Physiology Core (grant P30DK057521) directed by B.B. Kahn and O. Peroni for use of their equipment for the infusion experiment.

Address correspondence to: Mark A. Herman, Division of Endocrinology and Metabolism, Duke University Medical Center, 300 North Duke Street, Durham, North Carolina 27701, USA. Phone: 919.684.4005; E-mail: mark.herman@duke.edu.

MAH's present address is: Division of Endocrinology and Metabolism, Duke University Medical Center, Durham, North Carolina, USA.
1. Ford ES, Giles WH, Dietz WH. Prevalence of the metabolic syndrome among US adults: findings from the third National Health and Nutrition Examination Survey. JAMA 2002;287(3):356-359.

2. Brown MS, Goldstein JL. Selective versus total insulin resistance: a pathogenic paradox. Cell Metab. 2008;7(2):95-96.

3. Shimomura I, Matsuda M, Hammer RE, Bashmakov Y, Brown MS, Goldstein JL. Decreased IRS-2 and increased SREBP-1c lead to mixed insulin resistance and sensitivity in livers of lipodystrophic and ob/ob mice. Mol Cell. 2000;6(1):77-86.

4. Pajvani UB, Qiang L, Kangsamaksin T, Kitajewski J, Ginsberg HN, Accili D. Inhibition of Notch uncouples Akt activation from hepatic lipid accumulation by decreasing mTorc1 stability. Nat Med. 2013;19(8):1054-1060.

5. Li S, Brown MS, Goldstein JL. Bifurcation of insulin signaling pathway in rat liver: mTORC1 required for stimulation of lipogenesis, but not inhibition of gluconeogenesis. Proc Natl Acad Sci US A. 2010;107(8):3441-3446.

6. Taniguchi CM, Ueki K, Kahn R. Complementary roles of IRS- 1 and IRS- 2 in the hepatic regulation of metabolism. JClin Invest. 2005;115(3):718-727.

7. Samuel VT, et al. Mechanism of hepatic insulin resistance in non-alcoholic fatty liver disease. J Biol Chem. 2004;279(31):32345-32353.

8. Perry RJ, et al. Hepatic acetyl CoA links adipose tissue inflammation to hepatic insulin resistance and type 2 diabetes. Cell. 2015;160(4):745-758

9. Titchenell PM, et al. Direct hepatocyte insulin signaling is required for lipogenesis but is dispensable for the suppression of glucose produc- tion. Cell Metab. 2016;23(6):1154-1166.

10. Sindelar DK, Balcom JH, Chu CA, Neal DW, Cherrington $\mathrm{AD}$. A comparison of the effects of selective increases in peripheral or portal insulin on hepatic glucose production in the conscious dog. Diabetes. 1996;45(11):1594-1604.

11. Edgerton DS, et al. Insulin's direct effects on the liver dominate the control of hepatic glucose production. J Clin Invest. 2006;116(2):521-527.

12. Samuel VT, et al. Fasting hyperglycemia is not associated with increased expression of PEPCK or G6Pc in patients with type 2 diabetes. Proc Natl Acad Sci U S A. 2009;106(29):12121-12126.

13. Schwarz JM, Linfoot P, Dare D, Aghajanian K. Hepatic de novo lipogenesis in normoinsulinemic and hyperinsulinemic subjects consuming high-fat, low-carbohydrate and low-fat, high-carbohydrate isoenergetic diets. Am JClin Nutr. 2003;77(1):43-50.

14. Donnelly KL, Smith CI, Schwarzenberg SJ, Jessurun J, Boldt MD, Parks EJ. Sources of fatty acids stored in liver and secreted via lipoproteins in patients with nonalcoholic fatty liver disease. J Clin Invest. 2005;115(5):1343-1351.

15. Trinh KY, O’Doherty RM, Anderson P, Lange AJ, Newgard CB. Perturbation of fuel homeostasis caused by overexpression of the glucose-6-phosphatase catalytic subunit in liver of normal rats. J Biol Chem. 1998;273(47):31615-31620.

16. Vatner DF, et al. Insulin-independent regulation of hepatic triglyceride synthesis by fatty acids. Proc Natl Acad Sci U S A. 2015;112(4):1143-1148.

17. Agius L. High-carbohydrate diets induce hepatic insulin resistance to protect the liver from substrate overload. Biochem Pharmacol.
2013;85(3):306-312.

18. Otero YF, Stafford JM, McGuinness OP. Pathway-selective insulin resistance and metabolic disease: the importance of nutrient flux. J Biol Chem. 2014;289(30):20462-20469.

19. Haas JT, et al. Hepatic insulin signaling is required for obesity-dependent expression of SREBP-1c mRNA but not for feeding-dependent expression. Cell Metab. 2012;15(6):873-884.

20. Kotani K, Peroni OD, Minokoshi Y, Boss O, Kahn BB. GLUT4 glucose transporter deficiency increases hepatic lipid production and peripheral lipid utilization. JClin Invest. 2004;114(11):1666-1675.

21. Dentin R, et al. Liver-specific inhibition of ChREBP improves hepatic steatosis and insulin resistance in ob/ob mice. Diabetes. 2006;55(8):2159-2170.

22. Iizuka K, Bruick RK, Liang G, Horton JD, Uyeda K. Deficiency of carbohydrate response element-binding protein (ChREBP) reduces lipogenesis as well as glycolysis. Proc Natl Acad Sci US A. 2004;101(19):7281-7286.

23. Arden C, et al. Fructose 2,6-bisphosphate is essential for glucose-regulated gene transcription of glucose-6-phosphatase and other ChREBP target genes in hepatocytes. Biochem J. 2012;443(1):111-123.

24. Ma L, Robinson LN, Towle HC. ChREBP*Mlx is the principal mediator of glucose-induced gene expression in the liver. J Biol Chem. 2006;281(39):28721-28730.

25. Iizuka K, Miller B, Uyeda K. Deficiency of carbohydrate-activated transcription factor ChREBP prevents obesity and improves plasma glucose control in leptin-deficient (ob/ob) mice. Am J Physiol 
Endocrinol Metab. 2006;291(2):E358-E364.

26. Tran C, et al. Sex differences in lipid and glucose kinetics after ingestion of an acute oral fructose load. Br J Nutr. 2010;104(8):1139-1147.

27. Niewoehner CB, Gilboe DP, Nuttall GA, Nuttall FQ. Metabolic effects of oral fructose in the liver of fasted rats. Am J Physiol. 1984;247(4 Pt 1):E505-E512.

28. Malik VS, Popkin BM, Bray GA, Després JP, Willett WC, Hu FB. Sugar-sweetened beverages and risk of metabolic syndrome and type 2 diabetes: a meta-analysis. Diabetes Care. 2010;33(11):2477-2483.

29. Stanhope KL, et al. Consuming fructose-sweetened, not glucose-sweetened, beverages increases visceral adiposity and lipids and decreases insulin sensitivity in overweight/obese humans. JClin Invest. 2009;119(5):1322-1334.

30. Reaven GM. Banting lecture 1988. Role of insulin resistance in human disease. Diabetes. 1988;37(12):1595-1607.

31. Schwarz JM, et al. Effect of a high-fructose weight-maintaining diet on lipogenesis and liver fat. JClin Endocrinol Metab. 2015;100(6):2434-2442.

32. Erion DM, et al. The role of the carbohydrate response element-binding protein in male fructose-fed rats. Endocrinology. 2013;154(1):36-44.

33. Koo HY, Wallig MA, Chung BH, Nara TY, Cho BH, Nakamura MT. Dietary fructose induces a wide range of genes with distinct shift in carbohydrate and lipid metabolism in fed and fasted rat liver. Biochim Biophys Acta. 2008;1782(5):341-348.

34. Stamatikos AD, et al. Tissue specific effects of dietary carbohydrates and obesity on ChREBP $\alpha$ and ChREBP $\beta$ expression. Lipids. 2016;51(1):95-104.

35. Rodríguez-Calvo R, et al. Atorvastatin prevents carbohydrate response element binding protein activation in the fructose-fed rat by activating protein kinase A. Hepatology. 2009;49(1):106-115.

36. Herman MA, et al. A novel ChREBP isoform in adipose tissue regulates systemic glucose metabolism. Nature. 2012;484(7394):333-338.

37. Horton JD, Goldstein JL, Brown MS. SREBPs: activators of the complete program of cholesterol and fatty acid synthesis in the liver. JClin Invest. 2002;109(9):1125-1131.

38. Ludvik B, et al. A noninvasive method to measure splanchnic glucose uptake after oral glucose administration. JClin Invest. 1995;95(5):2232-2238.

39. Agius L. Glucokinase and molecular aspects of liver glycogen metabolism. Biochem J. 2008;414(1):1-18.

40. Poupeau A, Postic C. Cross-regulation of hepatic glucose metabolism via ChREBP and nuclear receptors. Biochim Biophys Acta. 2011;1812(8):995-1006.

41. Kawaguchi T, Takenoshita M, Kabashima T,
Uyeda K. Glucose and cAMP regulate the L-type pyruvate kinase gene by phosphorylation/

dephosphorylation of the carbohydrate response element binding protein. Proc Natl Acad Sci U S A. 2001;98(24):13710-13715.

42. Fukasawa M, Ge Q, Wynn RM, Ishii S, Uyeda K. Coordinate regulation/localization of the carbohydrate responsive binding protein (ChREBP) by two nuclear export signal sites: discovery of a new leucine-rich nuclear export signal site. Biochem Biophys Res Commun. 2010;391(2):1166-1169.

43. Benhamed F, et al. The lipogenic transcription factor ChREBP dissociates hepatic steatosis from insulin resistance in mice and humans. JClin Invest. 2012;122(6):2176-2194.

44. Davies MN, O'Callaghan BL, Towle HC. Glucose activates ChREBP by increasing its rate of nuclear entry and relieving repression of its transcriptional activity. J Biol Chem. 2008;283(35):24029-24038.

45. Li MV, Chen W, Poungvarin N, Imamura M, Chan L. Glucose-mediated transactivation of carbohydrate response element-binding protein requires cooperative actions from Mondo conserved regions and essential trans-acting factor 14-3-3. Mol Endocrinol. 2008;22(7):1658-1672.

46. Pfefferkorn JA, et al. Discovery of (S)-6-(3-cyclopentyl-2-(4-(trifluoromethyl)-1H-imidazol-1-yl) propanamido)nicotinic acid as a hepatoselective glucokinase activator clinical candidate for treating type 2 diabetes mellitus. J Med Chem. 2012;55(3):1318-1333.

47. Dentin R, et al. Glucose 6-phosphate, rather than xylulose 5-phosphate, is required for the activation of ChREBP in response to glucose in the liver. J Hepatol. 2012;56(1):199-209.

48. Nakae J, Kitamura T, Silver DL, Accili D. The forkhead transcription factor Foxo1 (Fkhr) confers insulin sensitivity onto glucose-6-phosphatase expression. JClin Invest. 2001;108(9):1359-1367.

49. Puigserver $P$, et al. Insulin-regulated hepatic gluconeogenesis through FOXO1-PGC-1alpha interaction. Nature. 2003;423(6939):550-555.

50. Matsumoto M, Pocai A, Rossetti L, Depinho RA, Accili D. Impaired regulation of hepatic glucose production in mice lacking the forkhead transcription factor Foxo1 in liver. Cell Metab. 2007;6(3):208-216

51. Kooner JS, et al. Genome-wide scan identifies variation in MLXIPL associated with plasma triglycerides. Nat Genet. 2008;40(2):149-151.

52. Johansen CT, Kathiresan S, Hegele RA. Genetic determinants of plasma triglycerides. J Lipid Res. 2011;52(2):189-206

53. Uyeda K, Repa JJ. Carbohydrate response element binding protein, ChREBP, a transcription factor coupling hepatic glucose utilization and lipid synthesis. Cell Metab. 2006;4(2):107-110. 54. Van Schaftingen E, Detheux M, Veiga da
Cunha M. Short-term control of glucokinase activity: role of a regulatory protein. FASEB $J$. 1994;8(6):414-419.

55. Van Schaftingen E. A protein from rat liver confers to glucokinase the property of being antagonistically regulated by fructose 6-phosphate and fructose 1-phosphate. Eur J Biochem. 1989;179(1):179-184

56. Filhoulaud G, Guilmeau S, Dentin R, Girard J, Postic C. Novel insights into ChREBP regulation and function. Trends Endocrinol Metab. 2013;24(5):257-268.

57. Eissing L, et al. De novo lipogenesis in human fat and liver is linked to ChREBP- $\beta$ and metabolic health. Nat Commun. 2013;4:1528.

58. Kursawe R, et al. Decreased transcription of ChREBP- $\alpha / \beta$ isoforms in abdominal subcutaneous adipose tissue of obese adolescents with prediabetes or early type 2 diabetes: associations with insulin resistance and hyperglycemia. Diabetes. 2013;62(3):837-844.

59. Lin HV, Accili D. Hormonal regulation of hepatic glucose production in health and disease. Cell Metab. 2011;14(1):9-19.

60. von Wilamowitz-Moellendorff A, et al. Glucose-6phosphate-mediated activation of liver glycogen synthase plays a key role in hepatic glycogen synthesis. Diabetes. 2013;62(12):4070-4082.

61. Dharaneeswaran H, et al. FOXO1-mediated activation of Akt plays a critical role in vascular homeostasis. Circ Res. 2014;115(2):238-251.

62. Humphreys SM, Frayn KN. Micro-method for preparing perchloric extracts of blood. Clin Chem. 1988;34(8):1657.

63. Zhu A, Romero R, Petty HR. An enzymatic fluorimetric assay for glucose-6-phosphate: application in an in vitro Warburg-like effect. Anal Biochem. 2009;388(1):97-101.

64. Folch J, Lees M, Sloane SGH. A simple method for the isolation and purification of total lipides from animal tissues. J Biol Chem. 1957;226(1):497-509.

65. Alegre M, Ciudad CJ, Fillat C, Guinovart JJ. Determination of glucose-6-phosphatase activity using the glucose dehydrogenase-coupled reaction. Anal Biochem. 1988;173(1):185-189.

66. Ramadoss $P$, et al. Novel mechanism of positive versus negative regulation by thyroid hormone receptor $\beta 1$ (TR $\beta 1$ ) identified by genome-wide profiling of binding sites in mouse liver. J Biol Chem. 2014;289(3):1313-1328.

67. Kim JK. Hyperinsulinemic-euglycemic clamp to assess insulin sensitivity in vivo. Methods Mol Biol. 2009;560:221-238.

68. Buescher JM, Moco S, Sauer U, Zamboni N. Ultrahigh performance liquid chromatography-tandem mass spectrometry method for fast and robust quantification of anionic and aromatic metabolites. Anal Chem. 2010;82(11):4403-4412. 